\title{
Improved Cauchy-characteristic evolution system for high-precision numerical relativity waveforms
}

\author{
Jordan Moxon® and Mark A. Scheel \\ Theoretical Astrophysics, Walter Burke Institute for Theoretical Physics, \\ California Institute of Technology, Pasadena, California 91125, USA \\ Saul A. Teukolsky \\ Theoretical Astrophysics, Walter Burke Institute for Theoretical Physics, \\ California Institute of Technology, Pasadena, California 91125, USA \\ and Cornell Center for Astrophysics and Planetary Science, Cornell University, \\ Ithaca, New York 14853, USA
}

(Received 6 July 2020; accepted 24 July 2020; published 28 August 2020)

\begin{abstract}
We present several improvements to the Cauchy-characteristic evolution procedure that generates highfidelity gravitational waveforms at $\mathcal{I}^{+}$from numerical relativity simulations. Cauchy-characteristic evolution combines an interior solution of the Einstein field equations based on Cauchy slices with an exterior solution based on null slices that extend to $\mathcal{I}^{+}$. The foundation of our improved algorithm is a comprehensive method of handling the gauge transformations between the arbitrarily specified coordinates of the interior Cauchy evolution and the unique (up to Bondi-Metzner-Sachs group transformations) BondiSachs coordinate system of the exterior characteristic evolution. We present a reformulated set of characteristic evolution equations better adapted to numerical implementation. In addition, we develop a method to ensure that the angular coordinates used in the volume during the characteristic evolution are asymptotically inertial. This provides a direct route to an expanded set of waveform outputs and is guaranteed to avoid pure-gauge logarithmic dependence that has caused trouble for previous spectral implementations of the characteristic evolution equations. We construct a set of Weyl scalars compatible with the Bondi-like coordinate systems used in characteristic evolution and determine simple, easily implemented forms for the asymptotic Weyl scalars in our suggested set of coordinates.
\end{abstract}

DOI: 10.1103/PhysRevD.102.044052

\section{INTRODUCTION AND MOTIVATION}

In the years since the original observations of the gravitational waves from compact binary mergers [1,2], sensitivity improvements of ground-based gravitational wave detectors have steadily increased the detection rate and the quality of data observed from merger events $[3,4]$. The detection and analysis of the resulting gravitational wave data require precise predictions of the signal from general relativity or beyond-GR theories. These predictions are used to extract faint gravitational wave signals from data contaminated by detector noise $[5,6]$ and to determine the detailed nature of the binary merger [7-11], including the parameters of the participating compact objects as well as constraints on parameters that describe deviations from general relativity $[12,13]$.

The three principal techniques for generating gravitational waveform predictions are post-Newtonian theory, self-force perturbation theory, and numerical relativity. Each of these techniques is most effective in a particular region of parameter space of a compact binary coalescence. Post-Newtonian theory is accurate and efficient when relativistic effects are small, so it models a binary at large separation well. Self-force techniques analytically expand the Einstein field equations in powers of the mass ratio, so they work well when describing a binary inspiral with disparate mass scales. Finally, numerical relativity is most useful when describing the strong-field region of comparable or intermediate mass ratio binary coalescence. In particular, the loudest part of the compact object coalescences observed by ground-based detectors aLIGO and VIRGO [5] are of the type for which numerical relativity simulations are most valuable. As the rate and extracted signal-to-noise ratio of gravitational wave detections improves, it will become increasingly important to have highly precise waveform predictions to make the best use of rich gravitational waveform data.

Multiple successful numerical relativity code bases have been constructed to determine the evolving metric of a compact binary coalescence [14-17]. Numerical relativity simulations operate by evolving Cauchy data on a finite spatial domain with approximate outgoing radiation boundary conditions. It is typically necessary to choose a gauge 
that makes the evolution stable and efficient, as opposed to choosing a gauge that provides convenient coordinates for interpreting the results. A key result of a successful Cauchy simulation is the spacetime metric and its derivatives on one or more timelike world tube surfaces at a chosen distance from the source, often $\sim 100-1000$ times the Schwarzschild radius of the compact companions.

The world tube data produced by a Cauchy simulation are then used as input to a separate calculation to determine the gravitational wave observables, such as the timedependent strain, asymptotically far from the system. The simplest method is to use linearized perturbation theory to derive an approximate asymptotic waveform by assuming that the nonlinear effects of general relativity are sufficiently small at the world tube to be neglected. A more precise method, known as waveform extrapolation, is to take advantage of the peeling theorem by computing the Weyl scalar $\psi_{4}$ at a sequence of concentric world tubes, fitting to a power series in $r^{-1}$, and using the $r^{-1}$ piece of $\psi_{4}$ to compute the asymptotic waveform $[18,19]$.

The most faithful method, however, is to take the world tube data provided by a Cauchy evolution as the inner boundary data for a second nonlinear evolution of the Einstein field equations. This second evolution is based on null (or "characteristic") hypersurfaces rather than spacelike hypersurfaces, and each null hypersurface extends fully to future null infinity $\mathcal{I}^{+}$.

This method of obtaining boundary data for the null foliation from an interior Cauchy evolution and propagating the gravitational wave information via an outer nonlinear evolution to $\mathcal{I}^{+}$is known as Cauchy-characteristic evolution $^{1}$ (CCE). Historically, the development of CCE has proceeded by first developing an evolution method on null hypersurfaces [20,21] and then creating a system for obtaining boundary data for the characteristic evolution determined by world tube data from a Cauchy evolution [22-26]. CCE was first implemented as a finite-difference code in Pitt Null [24,27], for which first results were presented in $[25,26]$. More recently, a spectral version of the algorithm was developed as a module in SpEC [28-31].

The CCE system evolves the Einstein field equations on a foliation of null hypersurfaces, each with an inner boundary on the timelike world tube provided by the Cauchy evolution. Introducing a compactified radial coordinate system allows the system to evolve on a finite spherical-shell domain with an outer boundary corresponding to $\mathcal{I}^{+}$. CCE is particularly powerful in determining

\footnotetext{
${ }^{1}$ The acronym CCE has also been used in the past to refer to "Cauchy-characteristic extraction," which describes only the part of the computation moving from the Cauchy coordinates to a set of quantities that could separately be evolved on null characteristic curves. Most of our descriptions refer to the entire algorithm as a single part of the wave computation, so we refer to the combination of Cauchy-characteristic extraction and characteristic evolution as simply CCE.
}

subdominant angular modes of the gravitational wave signal, including slowly varying "memory" modes, which are hoped to be detectable from aggregation of several merger observations. It is anticipated that modern advanced LIGO merger detections could reach the point where a memory signal is detectable with a signal to noise ratio of $\sim 5$ with $\sim 90$ detections similar to GW150914 [32].

A significant challenge in working with the CCE system is caused by the gauge of the metric data on the world tube, which is determined from the Cauchy evolution. The interior Cauchy evolution is typically forced to choose its coordinates to be convenient for timelike evolution in the strong-field region and is under no constraint to provide the world tube data in a gauge for which the coordinate representation of the metric falls off to a standard Minkowski form at $\mathcal{I}^{+}$. In practical implementations of the CCE system, it is frequently found that in the Cauchy evolution's gauge, all components of the metric that are not completely fixed by additional coordinate transformations possess nontrivial values at $\mathcal{I}^{+}$.

In the canonical treatment of the CCE system, the metric is put into a form that resembles the Bondi-Sachs form of the metric [see Eq. (1) below], but for which each of the metric components is constrained only to have finite value at $\mathcal{I}^{+}$rather than the precise falloff behavior [see Eq. (3) below] required by Bondi-Sachs coordinates. For the remainder of this paper, we will refer to the canonical CCE form of the metric as "Bondi-like" to distinguish those coordinates from a true Bondi-Sachs gauge. Our notation choices for the different forms of the metric are detailed in Sec. II.

The first implementation of CCE, PittNull [21], successfully evolved the characteristic system on the compactified asymptotic domain, but as a finite-difference implementation it was not efficient enough to be adopted ubiquitously [31]. The SpEC CCE implementation uses pseudospectral techniques to evolve the characteristic system, so it obtains precise waveforms far faster [28,30,31]. However, the precision of the SpEC spectral method is threatened by the development of pure-gauge logarithmic terms that arise in CCE implementations using Bondi-like coordinates. Such logarithmic dependence disrupts the exponential convergence with resolution otherwise enjoyed by spectral methods. Further, previous implementations of CCE have had the capability to extract only the asymptotic news and not other asymptotic quantities such as the Weyl scalars, because the expressions for those quantities are extremely complicated in general Bondi-like coordinates. Initial explorations with the SpEC CCE implementation [30] have demonstrated the feasibility of extracting the Weyl scalar $\psi_{4}$ from Bondi-Metzner-Sachs (BMS) flux quantities.

In this paper, we introduce a novel strategy for CCE that alleviates the most prominent remaining challenges for spectral implementations. Our new method is constructed from a well-chosen gauge restriction placed on the Bondilike coordinate system used to perform the evolution. 
Under our gauge restriction, the evolution system is provably free of pure-gauge logarithms, and all quantities needed to fix the gauge are easily calculated from the supplied Cauchy metric on the world tube. Further, our gauge restriction alleviates most of the complexity in evaluating the asymptotic waveform quantities, leading to simpler computation of the Bondi-Sachs news as well as succinct, practical formulas for the strain and for the leading contribution to all five Weyl scalars near $\mathcal{I}^{+}$.

The combination of mathematical results presented in this paper is best described by their role in the suggested algorithm for the next generation of CCE implementation:

(1) Initialize the characteristic system using well-chosen data on the initial null hypersurface that is compatible with a fully regular evolution procedure (thoroughly demonstrated in detail for the first time in Sec. V).

(2) At the intersection of the world tube and the initial null hypersurface, compute the Bondi-like metric from the supplied Cauchy metric on the world tube. This uses methods developed in [24] and reviewed in Sec. II.

(3) At the intersection of the world tube and the initial null hypersurface, compute the gauge transformation for a subset of the Bondi-Sachs spin-weighted scalars to a new "partially flat" gauge presented in this work (Sec. III). The partially flat gauge is easy to compute from the Bondi-like metric and provably avoids the pure-gauge logarithmic dependence (demonstrated in Sec. V) that arises in a general Bondi-like gauge.

(4) Perform the radial integration from the world tube to $\mathcal{I}^{+}$on the initial null hypersurface, for the first subset of Bondi-Sachs scalars determined in the previous substep. This determines these BondiSachs scalars everywhere on the initial null hypersurface. The radial differential equations for this step are improved in Sec. IV via a coordinate description optimized for practical implementation in a spectral domain. These equations are equivalent to a coordinate transformation and simplification applied to the original derivations [21].

(5) At the intersection of the initial null hypersurface and $\mathcal{I}^{+}$, use the results of the previous step to obtain information necessary to complete the gauge transformation to partially flat coordinates for the remaining Bondi-Sachs spin-weighted scalars that were not calculated in steps 3 and 4. (see the derivation of the volume coordinates in Sec. III and the newly developed procedure in Sec. V).

(6) At the intersection of the world tube and the initial null hypersurface, complete the transformation to partially flat coordinates for the final collection of Bondi-Sachs spin-weighted scalars (Sec. III), and perform the radial integration to obtain the remaining
Bondi-Sachs spin-weighted scalars everywhere on the initial null hypersurface.

(7) Perform an ordinary differential equation (ODE) integration in partially flat retarded time $u$ to obtain data on the next null hypersurface. This uses the Bondi-Sachs spin-weighted quantities obtained by steps 2-6.

(8) Repeat steps 2-7 for each successive null hypersurface.

(9) Use the simple expressions of the Weyl scalars in our new gauge (Sec. VI) to produce detailed gaugeinvariant (up to BMS freedom) information about the dynamic spacetime from the spin-weighted scalars evaluated in the evolution procedure.

The full detailed implementation strategy, complete with references to each of the equations for the computation steps, can be found in Sec. V C.

The numerical implementation of these techniques into an efficient, robust CCE code will be presented in forthcoming work [33]. The numerical implementation is built into the versatile code base SpECTRE [34], which targets scalable astrophysical simulation of various multiphysics systems, including multimessenger compact object coalescence. The efficient CCE code we are developing will be an important ingredient in the production of the gravitational wave predictions from astrophysical events simulated in SpECTRE.

\section{FOUNDATIONS OF CCE}

In this section, we review the current standard methods for CCE implementations. Thus, this section is entirely a recapitulation of calculations presented in past works $[21,24,28,35,36]$, adjusted to a notation compatible with the new calculations in the remaining sections of this paper. The CCE algorithm takes as input the spacetime metric and its derivatives on a chosen $(2+1)$-dimensional surface outside the strong-field region of the Cauchy simulation. The CCE procedure then adapts that metric to a Bondi-like form in which the Einstein field equations are amenable to a hierarchical evolution procedure. The metric components expressed as Bondi-like spin-weighted scalars are determined on each null hypersurface that intersects the world tube at constant Cauchy simulation time. A series of integrations along the null hypersurfaces generate the necessary data for performing a time step of the hyperbolic part of the characteristic system. The result of the characteristic evolution then specifies the full metric at $\mathcal{I}^{+}$, which is the outer boundary point of the compactified domain. Finally, that metric can be used in the evolution of inertial coordinates on $\mathcal{I}^{+}$that are used to produce meaningful gravitational wave observables in a BMS frame selected by the metric on the initial CCE hypersurface.

In the discussions of this section and throughout the $\mathrm{CCE}$ formalism, we find ourselves with an inconvenient surfeit of coordinate systems. To assist the reader in making sense 
of the various coordinates and indices, we include in Appendix A a table of the coordinates, their associated index adornment, and where they are used in the paper. All coordinate systems are considered spherical (possessing one timelike coordinate, one radial coordinate, and two coordinates on the sphere), Greek letters are used to indicate spacetime 4-indices, Latin letters in the range $i \cdots n$ are used to indicate spatial 3 -indices, and capital Latin letters are used to indicate angular 2-indices. Where necessary to clarify the coordinate dependence of spacetime fields, the symbols will also be decorated by the same adornment used for indices, such as $\stackrel{\circ}{\beta}$.

\section{A. Bondi-Sachs metric and Bondi-like coordinates for CCE}

In Bondi-Sachs coordinates, an asymptotically flat spacetime possesses a metric in spherical coordinates $\left\{\stackrel{\circ}{u}, \stackrel{\circ}{r}, \stackrel{\circ}{x}{ }^{\stackrel{\circ}{A}}\right\}$ of the form $[35,37,38]$

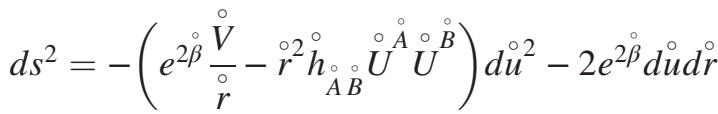

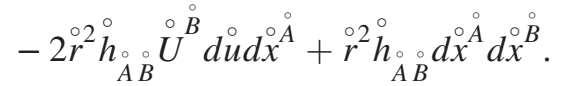

In these expressions, $\stackrel{\circ}{x}^{\circ}$ denotes the pair of angular coordinates. The Bondi-Sachs coordinates impose the gauge conditions $g_{r r}^{\circ}=0, g_{r \dot{\circ}}=0$, and the determinant of the angular components is set to the determinant of the unit sphere metric $q_{A B}^{\circ}$,

$$
\operatorname{det}\left(h_{\AA}^{\circ \circ}\right)=\operatorname{det}\left(q_{\AA}^{\circ}\right) .
$$

Further, the metric in Bondi-Sachs coordinates asymptotically approaches the Minkowski metric. The asymptotic restriction demands falloff rates for each of the metric components in (1) $[35,37,38]$ :

$$
\begin{aligned}
& \lim _{r \rightarrow \infty} \stackrel{\circ}{\beta}\left(\stackrel{\circ}{x}^{\circ}\right)=\mathcal{O}\left({ }^{\circ}-1\right), \\
& \lim _{r \rightarrow \infty} \stackrel{\circ}{V}\left(\stackrel{\circ}{\dot{x}^{\alpha}}\right)=\stackrel{\circ}{r}+\mathcal{O}\left(\stackrel{\circ}{r}^{0}\right), \\
& \left.\lim _{r \rightarrow \infty} \stackrel{\circ}{U} \stackrel{\stackrel{\circ}{A}}{\stackrel{\circ}{x}^{\circ}}\right)=\mathcal{O}\left({ }^{\circ}-2\right),
\end{aligned}
$$

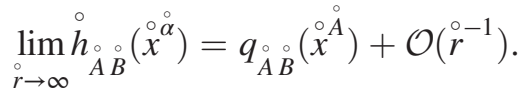

The Bondi-Sachs coordinate system is rather restrictive, and transforming to a Bondi-Sachs coordinate system from a generic metric depends on detailed information about the metric all the way out to $\mathcal{I}^{+}$(see Sec. III for a concrete description of how to obtain that information). For expedience, the standard methods for numerical implementations of CCE do not place the metric in a true Bondi-Sachs coordinate system, instead settling for the set of gauge conditions that can be evaluated locally in the bulk of the spacetime. The Bondi-like coordinate system used in previous CCE treatments takes the same form as Eq. (1),

$$
\begin{aligned}
d s^{2}= & -\left(e^{2 \beta} \frac{V}{r}-r^{2} h_{A B} U^{A} U^{B}\right) d u^{2}-2 e^{2 \beta} d u d r \\
& -2 r^{2} h_{A B} U^{B} d u d x^{A}+r^{2} h_{A B} d x^{A} d x^{B} .
\end{aligned}
$$

The gauge conditions imposed for (4) are the same local choices, $g_{r r}=0, \quad g_{r A}=0$, and $\operatorname{det}\left(h_{A B}\right)=\operatorname{det}\left(q_{A B}\right)$. However, the asymptotic dependence is relaxed to the simple requirement that all metric components are asymptotically finite,

$$
\begin{aligned}
& \lim _{r \rightarrow \infty} \beta\left(x^{\alpha}\right)=\mathcal{O}\left(r^{0}\right), \\
& \lim _{r \rightarrow \infty} W\left(x^{\alpha}\right)=\mathcal{O}\left(r^{0}\right), \\
& \lim _{r \rightarrow \infty} U^{A}\left(x^{\alpha}\right)=\mathcal{O}\left(r^{0}\right), \\
& \lim _{r \rightarrow \infty} h_{A B}\left(x^{\alpha}\right)=\mathcal{O}\left(r^{0}\right) .
\end{aligned}
$$

As an implementation note, the standard CCE algorithm does not even truly impose (5). Instead, the gauge fixed by most Cauchy world tube data is sufficiently well behaved to avoid pathological divergent dependence on the radius. Notably, requirements on Bondi-like coordinate systems are strictly weaker than the Bondi-Sachs coordinates, so any generic conclusions obtained about a Bondi-like metric can be immediately applied to a Bondi-Sachs metric.

To ease computations of the angular components of the metric, we introduce the complex dyad $q^{A}$ associated with the unit sphere metric $q_{A B}$. The following techniques may be applied in any coordinate system that satisfies the determinant condition $\operatorname{det}\left(h_{A B}\right)=\operatorname{det}\left(q_{A B}\right)$, so they are applicable to both Bondi-Sachs and Bondi-like coordinate systems. Here we use unadorned indices for notational simplicity. First,

$$
q_{A B}=\frac{1}{2}\left(q_{A} \bar{q}_{B}+\bar{q}_{A} q_{B}\right) .
$$

Note that under this definition, the dyad has normalization $q^{A} \bar{q}_{A}=2$, which is chosen for the numerical convenience of avoiding factors of $\sqrt{2}$ in dyad components and derivatives. For computations in which the angular components must be expanded explicitly, this paper will make use of standard spherical coordinate angles $\{\theta, \phi\}$ and select the complex dyad 


$$
q^{A}=\left\{-1, \frac{-i}{\sin \theta}\right\}
$$

Each angular component of the Bondi-like metric is contracted with either the complex dyad $q^{A}$ or its conjugate $\bar{q}^{A}$ to form spin-weighted scalar components $[21,36]$. The spin weight of the scalars is determined by how they transform according to rotations in the choice of complex dyad $q^{A}$. The unit sphere metric is symmetric under inplane rotations of the complex dyad

$$
q^{A} \rightarrow q^{A} e^{i \psi}
$$

We then refer to a quantity $v$ as possessing a spin weight $s$ if it transforms as

$$
v \rightarrow v e^{i s \psi}
$$

under the dyad rotation (8). Consequently, for any angular vector $v^{A}$, the scalar $v=v^{A} q_{A}$ is of spin weight 1 , and $\bar{v}=v^{A} \bar{q}_{A}$ is of spin weight -1 . We adopt the following standard notation for spin-weighted scalars derived from angular Bondi-like metric components:

$$
\begin{array}{cc}
U \equiv U^{A} q_{A} & (\text { spin weight } 1), \\
Q \equiv r^{2} e^{-2 \beta} q^{A} h_{A B} \partial_{r} U^{B} & (\text { spin weight } 1), \\
r^{2} W \equiv V-r & (\text { spin weight } 0), \\
J \equiv \frac{1}{2} q^{A} q^{B} h_{A B} & (\text { spin weight } 2), \\
K \equiv \frac{1}{2} q^{A} \bar{q}^{B} h_{A B} & \\
=\sqrt{1-J \bar{J}} & (\text { spin weight } 0),
\end{array}
$$

where $Q$ in (10b) is introduced to reduce the Einstein field equations for the spin-weighted components to only first derivatives in $r$. The equality in (10e) arises from the normalization of the angular metric determinant $\operatorname{det}\left(h_{A B}\right)=\operatorname{det}\left(q_{A B}\right)$ from the Bondi-like construction.

We introduce the spin-weighted angular differential operators $ð$ and $\bar{\partial}$. For any spin-weighted scalar quantity $v=q_{1}^{A_{1}} \cdots q_{n}^{A_{n}} v_{A_{1} \cdots A_{n}}$, where each $q_{i}$ may be either $q$ or $\bar{q}$, we define the spin-weighted derivatives,

$$
\begin{aligned}
& ð v=q_{1}^{A_{1}} \cdots q_{n}^{A_{n}} q^{B} D_{B} v_{A_{1} \cdots A_{n}}, \\
& \bar{ð} v=q_{1}^{A_{1}} \cdots q_{n}^{A_{n}} \bar{q}^{B} D_{B} v_{A_{1} \cdots A_{n}} .
\end{aligned}
$$

In (11), $D_{A}$ denotes the covariant derivative associated with the unit sphere metric $q_{A B}$. In particular, for our choice of the complex dyad (7), the spin-weighted derivative operator applied to a scalar $v$ of spin weight $s$ takes the coordinate form

$$
ð v=-(\sin \theta)^{s}\left(\frac{\partial}{\partial \theta}+\frac{i}{\sin \theta} \frac{\partial}{\partial \phi}\right)\left[(\sin \theta)^{-s} v\right] .
$$

\section{B. Boundary transformations}

The first task of a numerical implementation of CCE is to transform the metric provided by a Cauchy simulation on a particular world tube to the Bondi-like form (4). In this section, we review the practical steps that can be used to impose the gauge conditions $g_{r r}=0, g_{r A}=0, \operatorname{det}\left(g_{A B}\right)=$ $\operatorname{det}\left(q_{A B}\right)$ on the input world tube $\Gamma$. The material we review here is similar to the procedures developed in [24], though we describe the streamlined procedure [31] that does not first extrapolate to a surface of constant Bondi-like $r$. Instead, the initial data in our description are given at a surface described by a world tube radius function $r=R\left(x^{A}\right)$.

The input to the CCE algorithm is a set of metric components $g_{\alpha^{\prime} \beta^{\prime}}$ and their first partial derivatives $g_{\alpha^{\prime} \beta^{\prime} \gamma^{\prime}}$ on a surface $S_{r^{\prime}}$ of constant $r^{\prime}$ and $t^{\prime}$ from the Cauchy evolution. On that surface, we perform the Arnowitt-DeserMisner (ADM) decomposition of the metric components natural for the Cauchy evolution:

$$
\begin{aligned}
d s^{2}= & \left(-\alpha^{2}+\beta^{i^{\prime}} \beta j^{j^{\prime}} g_{i^{\prime} j^{\prime}}\right) d t^{\prime 2} \\
& +2 \beta^{i^{\prime}} g_{i^{\prime} j^{\prime}} d x^{\prime j^{\prime}} d t^{\prime}+g_{i^{\prime} j^{\prime}} d x^{\prime i^{\prime}} d x^{\prime j^{\prime}} .
\end{aligned}
$$

Here $\beta^{i^{\prime}}$ is the shift vector, which is not to be confused with the metric component $\beta$ in the Bondi-like metric (4).

First, we wish to construct a null vector $l^{\alpha^{\prime}}$ to act as the generators for the outgoing null cone at the world tube surface. The normal vector $s^{\alpha^{\prime}}$ to the provided surface $S_{r^{\prime}}$ is used for the radial component for a candidate null vector,

$$
\begin{aligned}
& s^{t^{\prime}}=0, \\
& s^{i^{\prime}}=\frac{g^{i^{\prime} j^{\prime}} \partial_{i^{\prime}} r^{\prime}}{\sqrt{g^{i^{\prime} j^{\prime}} \partial_{i^{\prime}} r^{\prime} \partial_{j^{\prime}} r^{\prime}}} .
\end{aligned}
$$

Define also the hypersurface normal associated with the Cauchy evolution $n^{\alpha^{\prime}}$,

$$
\begin{aligned}
& n^{t^{\prime}}=\frac{1}{\alpha}, \\
& n^{i^{\prime}}=\frac{-\beta^{i^{\prime}}}{\alpha} .
\end{aligned}
$$

Then, by construction, $n^{\alpha^{\prime}} n_{\alpha^{\prime}}=-1, s^{\alpha^{\prime}} s_{\alpha^{\prime}}=1$, and $n^{\alpha^{\prime}} s_{\alpha^{\prime}}=0$. Therefore, we determine a normalized null vector $l^{\alpha^{\prime}}$ as [24] 


$$
l^{\alpha^{\prime}}=\frac{n^{\alpha^{\prime}}+s^{\alpha^{\prime}}}{\alpha-g_{i^{\prime} j^{\prime}} \beta^{i^{\prime}} s^{j^{\prime}}} .
$$

We now construct a new set of null-radius coordinates $\{\underline{u}, \underline{\lambda}, \underline{x} \underline{A}\}$, where $\underline{\lambda}$ is an affine parameter along the null rays generated by $l^{\alpha^{\prime}}$. In the set of null-radius coordinates, we use the time and angular coordinates from the Cauchy data unchanged $\underline{x}^{A}=\delta^{\underline{A}}{ }_{A^{\prime}} x^{\prime A^{\prime}}, \underline{u}=t^{\prime}$.

The metric components in the null-radius coordinates are

$$
\begin{aligned}
g_{\underline{\lambda} \underline{u}} & =l^{\alpha^{\prime}} g_{\alpha^{\prime} t^{\prime}}=-1, \\
g_{\underline{\lambda} \underline{\lambda}} & =l^{\alpha^{\prime}} l^{\beta^{\prime}} g_{\alpha^{\prime} \beta^{\prime}}=0, \\
g_{\underline{\lambda} \underline{A}} & =l^{\alpha^{\prime}} \frac{\partial x^{\prime i^{\prime}}}{\partial \underline{x} \underline{A}} g_{\alpha^{\prime} i^{\prime}}=l^{\alpha^{\prime}} \delta_{\underline{A}}^{A^{\prime}} \frac{\partial x^{i^{\prime}}}{\partial x^{\prime A^{\prime}}} g_{\alpha^{\prime} i^{\prime}}=0, \\
g_{\underline{u} \underline{u}} & =g_{t^{\prime} t^{\prime}}, \\
g_{\underline{u}} \underline{A} & =\frac{\partial x^{\prime i^{\prime}}}{\partial \underline{x}^{\underline{A}}} g_{t^{\prime} i^{\prime}}=\delta_{\underline{A}}^{A^{\prime}} \frac{\partial x^{\prime i^{\prime}}}{\partial x^{\prime A^{\prime}}} g_{t^{\prime} t^{\prime}}, \\
g_{\underline{A} \underline{B}} & =\frac{\partial x^{\prime i^{\prime}}}{\partial \underline{x}^{\underline{A}}} \frac{\partial x^{\prime j^{\prime}}}{\partial \underline{x}^{\underline{B}}} g_{i^{\prime} j^{\prime}} \\
& =\delta_{\underline{A}}^{A^{\prime}} \delta_{\underline{B}}^{B^{\prime}} \frac{\partial x^{i^{\prime}}}{\partial x^{\prime A^{\prime}}} \frac{\partial x^{i^{\prime}}}{\partial x^{\prime B^{\prime}}} g_{i^{\prime} j^{\prime}} .
\end{aligned}
$$

At this point, we have determined a suitable null coordinate system for the world tube metric, enforcing $g_{\lambda \lambda}=g_{\lambda A}=0$. To complete the transformation to coordinates compatible with the Bondi-like metric form (4), we must construct an areal radial coordinate $r$ such that $g_{A B}=r^{2} h_{A B}$ and $\operatorname{det}\left(h_{A B}\right)=\operatorname{det}\left(q_{A B}\right)$.

Define the Bondi-like radius,

$$
r=\left[\frac{\operatorname{det}\left(g_{\underline{A} \underline{B}}\right)}{\operatorname{det}\left(q_{\underline{A}}\right)}\right]^{1 / 4}
$$

where, as in the previous subsection, $q_{\underline{A} \underline{B}}$ represents the angular metric on the unit sphere. The Bondi-like coordinates adopted by the standard characteristic extraction algorithm are $\left\{r, u, x^{A}\right\}$, where the time and angular coordinates are again unchanged from the original set determined by the Cauchy evolution $u=\underline{u}, x^{A}=\delta^{A}{ }_{A} \underline{x} \underline{A}$.

Finally, the form of the Bondi-like metric (4) may be used to determine the spin-weighted scalars associated with the transformed metric. The Bondi-like scalars are most conveniently determined from the up-index components of the metric [24,31]

$$
\begin{aligned}
& \beta=-\frac{1}{2} \ln \left(-g^{u r}\right)=-\frac{1}{2} \ln \left(\partial_{\underline{\lambda}} r\right), \\
& U=\frac{g^{u A}}{g^{u r}} q_{A},
\end{aligned}
$$

$$
\begin{aligned}
& W=\frac{1}{r}\left(1-\frac{g^{r r}}{g^{u r}}\right), \\
& J=-\frac{1}{2} r^{2} q_{A} q_{B} g^{A B}, \\
& K=\sqrt{1+J \bar{J}}, \\
& Q=r^{2}\left(J \partial_{\underline{\lambda}} \bar{U}+K \partial_{\underline{\lambda}} U\right) .
\end{aligned}
$$

Here the up-index components of the metric $g^{\mu \nu}$ can be determined from Eqs. (17) and the coordinate transformation from $x^{\mu}$ to $x^{\mu}$; for details see [31]. Note that several terms in Eqs. (19) seem to depend on derivatives of the Jacobian $\partial x^{\mu} / \partial x^{\nu}$, and so would have the danger of requiring multiple derivatives of the input metric. In fact, the characteristic extraction algorithm depends only on first derivatives of the input metric. To make the ability to represent all requisite quantities in terms of first derivatives explicit, note the identities from [31]

$$
\begin{aligned}
U_{, \underline{\lambda}}= & -\left(g^{\underline{\lambda} \underline{A}_{, \underline{\lambda}}}+\frac{r_{, \underline{\lambda}} \underline{B}}{r_{, \underline{\lambda}}} g^{\underline{A} \underline{B}}+\frac{r}{, \underline{B}}_{r_{\underline{\lambda}}} g^{\underline{A}} \underline{B}\right) \\
& +2 \beta_{, \underline{\lambda}}\left(U+g^{\underline{A}} \underline{\underline{A}}_{\underline{A}}\right), \\
J_{, \underline{\lambda}}= & -\frac{1}{2} r^{2} q_{\underline{A}} q_{\underline{B}} h^{\underline{A}} \underline{B}_{, \underline{\lambda}}-\frac{2 r_{, \underline{\lambda}}}{r} J .
\end{aligned}
$$

The term $\beta_{, \lambda}$ (which otherwise would depend on the second derivative $r_{, \underline{\lambda}}$ ) can be written in terms of only first derivatives of the input metric by using one of the components of the Einstein field equations:

$$
\beta_{, \underline{\lambda}}=\frac{r}{8 r_{, \underline{\lambda}}}\left(J_{, \underline{\lambda}} \bar{J}_{, \underline{\lambda}}+\left(K_{, \underline{\lambda}}\right)^{2}\right)
$$

Finally, we define the helpful quantity $H \equiv \partial_{u} J$ on the world tube:

$$
H \equiv J_{, u}=-\frac{1}{2} r^{2} q_{\underline{A}} q_{\underline{B}} h^{A} \underline{B}_{, \underline{u}}-\frac{2 r_{, \underline{u}}}{r} J .
$$

We emphasize that the set of local computations summarized in this section is sufficient to enforce the Bondilike gauge conditions $g_{r r}=g_{r A}=0$ and $\operatorname{det} g_{A B}=\operatorname{det} q_{A B}$, but enforces no restriction on the asymptotic dependence of the metric components. In general, the metric in these Bondi-like coordinates will not asymptotically approach a Minkowski metric, and therefore quantities determined at $\mathcal{I}^{+}$will be in a noninertial gauge and require an additional correction described below in Sec. II D. 


\section{Hierarchical evolution system}

The characteristic evolution proceeds in ascending retarded time $\bar{u}$, computing the Bondi-like metric on $\bar{u}=$ const null hypersurfaces (see Fig. 1). The inputs to this evolution system are the value of $J$ on the initial hypersurface and the spin-weighted scalars $\beta, U, Q, W$, and $H$ on a world tube $\Gamma$, as determined by the computation reviewed in Sec. II B. The alignment of the characteristic extraction spacetime foliation with the outgoing null rays and the choice of a convenient combination of independent Einstein field equation components ensures that the only Bondi-like spin-weighted scalar that needs be evolved between the hypersurfaces is $J$. The remaining metric quantities $\beta, U, Q, W$, and $H$ determined on each hypersurface instead satisfy purely spatial partial differential equations, which are hereafter and in the literature referred to as the set of hypersurface equations.

The Einstein field equations for the Bondi-like metric (4) result in the following hypersurface equations, which take a computationally convenient hierarchical form:

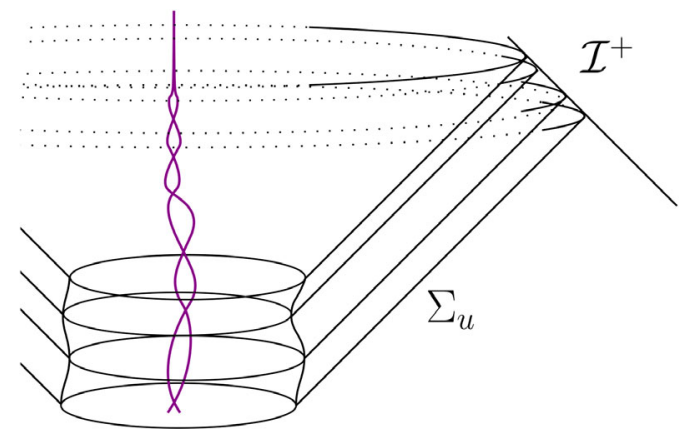

FIG. 1. The Cauchy-characteristic extraction and characteristic evolution computational domain. The central circular region represents the finite spherical domain of the Cauchy code. $\mathrm{CCE}$ is evaluated on the null surfaces extending from the input world tube to $\mathcal{I}^{+}$. Numerical implementations take advantage of a compactified radial coordinate to solve for the asymptotic field in a finite numerical domain. The characteristic hypersurface equations, Eqs. (23), determine the field values along each $\Sigma_{u}$ using boundary values on the world tube. The evolution equation determines the remaining degrees of freedom on subsequent hypersurfaces.

$$
\begin{gathered}
\beta_{, r}=S_{\beta}(J), \\
\left(r^{2} Q\right)_{, r}=S_{Q}(J, \beta), \\
U_{, r}=S_{U}(J, \beta, Q), \\
\left(r^{2} W\right)_{, r}=S_{W}(J, \beta, Q, U), \\
(r H)_{, r}+L_{H}(J, \beta, Q, U, W) H+L_{\bar{H}}(J, \beta, Q, U, W) \bar{H}=S_{H}(J, \beta, Q, U, W) .
\end{gathered}
$$

The form of the system (23) allows for the technique of first determining $J$ on a hypersurface $\Sigma_{u}$ of constant $u$, then using the value of $J$ and the world tube boundary value $\left.\beta\right|_{\Gamma}$ to determine $\beta$ on the hypersurface $\Sigma_{u}$ via (23), and following the cascaded equations in order to obtain $H$. Finally, $\partial_{u} J=H$, so once $H$ is determined by the hypersurface equations, it may be used to step $J$ to the next hypersurface. Because $\partial_{u} J$ can thus be considered a (very complicated) function of only $J$, plus boundary data that depend on $u$, the stepping in $u$ can be done using any ODE integration method.

The full form for each equation in (23) may be found in a variety of coordinate systems in prior derivations [21,28] and is rederived in the accompanying Mathematica notebook [39]. However, practical implementations in spectral coordinates require an additional coordinate transformation from previously published results, and the expressions can be streamlined for numerical use by using identities between the Bondi-like spin-weighted scalars. In Sec. IV, we present a simplified set of hypersurface equations tailored to direct numerical implementation.

\section{Observables at $\mathcal{I}^{+}$}

The final stage of the previously developed CCE algorithm is to determine the gravitational-wave observables from the Bondi-like spin-weighted scalars derived from the evolution scheme described in the previous Sec. II C. The primary gravitational-wave observable we consider is the Bondi news. In a true Bondi-Sachs coordinate system (1), it is defined as the leading part of the first time derivative of the angular metric:

$$
N_{A B}=\lim _{r \rightarrow \infty}\left(\stackrel{\circ}{r} \partial_{u} h_{\AA B}^{\circ}\right) .
$$

When working with the spin-weighted metric quantities, the same information may be conveyed by expressing the news as a spin-weighted scalar,

$$
N=\frac{1}{2} \stackrel{\circ}{\stackrel{q}{q} \stackrel{\circ}{\bar{q}}{ }^{\circ} N_{A B} \circ}=\lim _{r \rightarrow \infty}\left(\stackrel{\circ}{r} \partial_{u}^{\circ} J\right)
$$


Importantly, while the inputs to computing the BondiSachs news $N$ depend on the coordinate system, it is a gauge-invariant (up to the residual BMS freedom) quantity defined such that in every coordinate system it takes the value computed by the formula (24) in the Bondi-Sachs coordinates. See $[21,40]$ for alternative formulas for defining the same Bondi news.

Previous treatments of the characteristic evolution systems then face the challenge of computing the spinweighted Bondi news function $N\left(u, x^{A}\right)$ in a Bondi-like frame by analytically computing a form for the news that holds for a generic Bondi-like gauge [21]. That form of the news is then computed using the asymptotic values of the spin-weighted scalars determined by the hypersurface equations (23). However, the Bondi-like form of the news does not compensate for the fact that the hypersurface equations have been solved in coordinates that are not asymptotically inertial.

To account for the coordinate change at $\mathcal{I}^{+}$, the CCE algorithm then computes the set of inertial coordinates

$\left\{\stackrel{\circ}{u}\left(u, x^{A}\right), \stackrel{\circ}{x}^{A}\left(u, x^{A}\right)\right\}$ on $\mathcal{I}^{+}$by solving the set of geodesic equations [21],

$$
\begin{aligned}
\partial_{u}{ }^{\circ} x^{\circ} & =-U^{B} \partial_{B}{ }^{\circ} x^{\circ}, \\
\partial_{u} \stackrel{\circ}{u} & =\left(-U^{B} \partial_{B} \stackrel{\circ}{u}+1\right) e^{2 \beta},
\end{aligned}
$$

which may be integrated in $u$ along $\mathcal{I}^{+}$. Finally, the news function derived from Bondi-like quantities may be evaluated in terms of the inertial coordinates $N\left(u, x^{A}\right)$. This final result is then equivalent to the news that would have been computed from the simpler form (24) if the system had been in a true Bondi-Sachs gauge from the start. The news in the inertial coordinates is then the primary gravitational waveform observable provided by the CCE algorithm.

In previous work, the coordinate transformation accomplished by Eq. (26) was considered only at $\mathcal{I}^{+}$. In Sec. III below, we make use of a modified and streamlined version of the same angular coordinate transformation to derive a complete set of new transformations for the Bondi-like scalars throughout the bulk of the spacetime. Additionally, we extend the derivation to provide a set of new coordinate conditions sufficient to place the bulk spacetime metric in the Bondi-Sachs coordinates, which are then uniquely gauge-fixed up to BMS transformations. Taken together with the prior results [24] summarized in Sec. II B, the technique given in Sec. III describes a concrete method to transform any input metric to Bondi-Sachs coordinates. Further, we derive an alternative form for the news function in an arbitrary Bondi-like gauge that is compatible with previous results, but somewhat simpler to compute.

The results of Sec. III below also grant the freedom to apply the coordinate transformations at any point during the CCE algorithm. We show in Sec. V that there is a significant numerical advantage to performing a portion of those coordinate transformations on the world tube boundary data before the characteristic evolution. The use of the world tube coordinate transformation provably avoids puregauge logarithmic dependence that has previously plagued spectral implementations of CCE [28].

\section{TRANSFORMATIONS FROM BONDI-LIKE TO BONDI COORDINATES}

In this section, we present a sequence of explicit coordinate transformations that take any Bondi-like metric (4) to a true Bondi-Sachs frame, in which the metric asymptotically approaches the Minkowski metric at the appropriate rates determined in [35] and reviewed in Eqs. (3). We refer to a new intermediate gauge derived in these steps as the "partially flat" gauge. This gauge is well suited to numerical implementations and provably avoids pure-gauge logarithms (see Sec. V). Enforcing the partially flat gauge underlies many of the improvements we derive in this paper for practical CCE implementations.

It is also worth noting that the transformations presented here are of general interest to any computation for which it is desirable to determine a highly fixed gauge from an input metric in arbitrary coordinates, such as self-force calculations [41,42] or detailed comparisons between general relativity computational techniques. Starting from ADM quantities on a spherical 2-surface, the standard Cauchy-characteristic extraction descriptions from [24] will determine a metric in an arbitrary Bondi-like gauge, which is not yet unique up to BMS transformations because of the significant freedom in asymptotic behavior. The transformations in this section complete the procedure, giving a concrete method to determine the true Bondi-Sachs metric, unique up to BMS transformations, for the spacetime bulk. We present the final transformation both as a partial differential equation that may be solved for the exact Bondi-Sachs coordinates and as an expansion near $\mathcal{I}^{+}$.

The coordinate transformations presented here not only improve the gauge of practical computations, but also supply the metric components in a Bondi-Sachs frame. These metric components are easily related to asymptotic waveform quantities, such as the news (24) and the Weyl scalars discussed in Sec. VI below. Our form for the news is compatible with earlier computations of the same quantity by other methods $[21,40]$.

\section{A. Step-by-step coordinate transformations}

For the detailed presentation of the coordinate transformations, it is convenient to work with the up-index form of the Bondi-like metric, as it more easily maps to the transformations of the spin-weighted scalars $\{\beta, W, U, J\}$. Further, we adopt the radial coordinate $l=1 / r$, which is convenient for asymptotic expansions. In cases where the 
only coordinate alteration is the use of the inverse radial coordinate, we choose not to introduce new index embellishments to avoid overcomplicating the notation. The upindex metric in the Bondi-like form (4) is

$$
g^{\mu \nu}=\left[\begin{array}{ccc}
0 & l^{2} e^{-2 \beta} & 0 \\
l^{2} e^{-2 \beta} & l^{3} e^{-2 \beta}(W+l) & l^{2} e^{-2 \beta} U^{A} \\
0 & l^{2} e^{-2 \beta} U^{B} & l^{2} h^{A B}
\end{array}\right] .
$$

The procedure for completely establishing a volume Bondi-Sachs metric is accomplished by the following sequence of steps:

(1) Establish asymptotically inertial angular coordinates $\hat{x}^{\hat{A}}\left(u, x^{A}\right)$, removing the asymptotically constant part of $U^{A}$.

(2) Modify the radial coordinate to $\hat{l}\left(l, u, x^{A}\right)$ to enforce the desired determinant in the angular block of the metric. Following this step, the coordinate system is the partially flat coordinates $\left\{\hat{u}=u, \hat{l}, \hat{x}^{\hat{A}}\right\}$ we have referred to previously.

(3) Alter the time coordinate to the asymptotically inertial $\stackrel{\circ}{u}$, removing the asymptotically constant part of $\beta^{(0)}$. This will, however, disrupt the Bondi form of the metric, giving an $\mathcal{O}\left(\hat{l}^{2}\right)$ contribution to $g^{i \hat{A}}$.

(4) Impose the Bondi metric restriction $g^{u A}=0$ by introducing a set of angular coordinates modified only at subleading order in $\hat{l}, \stackrel{\circ}{x}=\hat{x}^{\hat{A}}+$ $l_{x}^{\circ}(1)\left(u, l, x^{A}\right)$.

(5) Restore the Bondi-Sachs coordinate conditions by constructing an areal radius $\stackrel{\circ}{l}$ associated with $\stackrel{\circ}{x}^{\circ}$, again modifying only the subleading part in $\hat{l}$.

Following this step, the coordinates $\left\{\stackrel{\circ}{u}, \stackrel{\circ}{l}, \stackrel{\circ}{x} \stackrel{\circ}{x}^{\prime}\right\}$ are in the Bondi-Sachs frame, fixed up to BMS transformations.

Following steps 2 and 5, we will present the metric in terms of the original Bondi-like spin-weighted scalar components. For simplicity of presentation, we do not do so for the metric following steps 1,3 , and 4 , as the intermediate metric at those stages of the computation is not even in Bondi-like form, so it has limited utility.

We note that our set of coordinate transformations from steps $1-5$ is in agreement with the set of inertial coordinates on $\mathcal{I}^{+}$established by [21], but possesses additional radial dependence necessary to enforce an asymptotically flat Bondi metric that is also valid in the bulk of the spacetime. The conditions we impose are equivalent to the coordinate conditions previously explicated for the Bondi-Sachs metric [43], but our derivation gives rise to an important new intermediate gauge and produces explicit recipes for the Bondi-Sachs quantities from arbitrary Bondi-like inputs.
Following the program laid out above, step 1 selects a set of angular coordinates $\hat{x}^{\hat{A}}$ such that

$$
\partial_{u} \hat{x}^{\hat{A}}=-\partial_{A} \hat{x}^{\hat{A}} U^{(0) A},
$$

where $U^{(0) A}$ is the asymptotic part of $U^{A}$ defined by the expansion

$$
U^{A}=U^{(0) A}\left(u, x^{A}\right)+l U^{(R) A}\left(u, l, x^{A}\right) .
$$

In this expansion the superscript $(R)$ is used to indicate the "remainder" after the subtraction of the asymptotically constant $U^{(0)}$. Note that (28) can be numerically evaluated using standard time-integration methods, and permits arbitrary initial angular coordinates $\hat{x}^{\hat{A}}(u=0)$. We choose a natural initial condition for this time integration, which is to take $x^{\hat{A}}\left(u=0, x^{A}\right)=\delta^{\hat{A}}{ }_{A} x^{A}$. Note that $l$ is no longer the areal radius for the new set of angular coordinates. Therefore, the intermediate metric between step 1 and step 2 is not Bondi-like, so it could not be used in a characteristic evolution without rederiving the equations of motion.

In step 2 we restore the Bondi-like form by performing a transformation to an areal inverse radius $\hat{l}$ for the new set of angular coordinates $\hat{x}^{\hat{A}}$. The new areal coordinate is determined by the alteration of the angular determinant:

$$
\hat{l}=l \sqrt{\operatorname{det}\left(\partial_{A} \hat{x}^{\hat{A}}\right)}\left(\frac{\operatorname{det}\left(\hat{q}_{\hat{A} \hat{B}}\right)}{\operatorname{det}\left(q_{A B}\right)}\right)^{1 / 4} \equiv \frac{l}{\hat{\omega}\left(u, x^{A}\right)} .
$$

To more simply express the transformations of the spinweighted scalars following this coordinate transformation, we introduce the spin-weighted Jacobian factors,

$$
\begin{aligned}
& \hat{a}=\hat{q}^{\hat{A}} \partial_{\hat{A}} x^{A} q_{A}, \\
& \hat{b}=\hat{\bar{q}}^{\hat{A}} \partial_{\hat{A}} x^{A} q_{A} .
\end{aligned}
$$

Under these definitions, we have the convenient identity for the conformal factor $\hat{\omega}$ :

$$
\hat{\omega}=\frac{1}{2} \sqrt{\hat{b} \hat{\bar{b}}-\hat{a} \hat{\bar{a}}}
$$

After steps 1 and 2, the new spin-weighted scalar components of the metric are

$$
\begin{aligned}
\hat{\beta}= & \beta-\frac{1}{2} \log \hat{\omega}, \\
\hat{J}= & \frac{\hat{b}^{2} J+\hat{a}^{2} \bar{J}+2 \hat{a} \hat{\bar{b}} K}{\hat{\omega}^{2}}, \\
\hat{U}= & \frac{1}{2 \hat{\omega}^{2}}\left(\hat{\bar{b}}\left(U-U^{(0)}\right)-\hat{a}\left(\bar{U}-\bar{U}^{(0)}\right)\right) \\
& -\frac{\hat{l}^{2 \hat{\beta}}}{\hat{\omega}}(\hat{\partial} \hat{\omega} \hat{K}-\hat{\widehat{\partial}} \hat{\omega} \hat{J}),
\end{aligned}
$$




$$
\begin{aligned}
\hat{W}= & W+(\hat{\omega}-1) \hat{l}-\frac{2 \partial_{\hat{u}} \hat{\omega}}{\hat{\omega}} \\
& +\frac{e^{2 \hat{\beta}} \hat{l}}{2 \hat{\omega}^{2}}\left(\hat{\bar{\partial}} \hat{\omega}^{2} \hat{J}+\hat{\partial} \hat{\omega}^{2} \hat{\bar{J}}\right) .
\end{aligned}
$$

The hypersurface equations for $W$ and $\hat{W}$ or expansion of derivatives in the determinant (30) may be used to infer $\partial_{\hat{u}} \hat{\omega}$ :

$$
\begin{aligned}
\partial_{\hat{u}} \hat{\omega} & =\frac{\hat{\omega}}{4}\left(\hat{\jmath} \overline{\mathcal{U}}^{(0)}+\hat{\bar{\partial} \mathcal{U}^{(0)}}\right), \\
\mathcal{U}^{(0)} & \equiv \frac{1}{2}\left(\hat{\bar{b}} U^{(0)}-\hat{a} \bar{U}^{(0)}\right) .
\end{aligned}
$$

Our choice to use angular coordinates $\hat{x}^{\hat{A}}$ that initially coincide with the Cauchy angular coordinates $x^{A}$ ensures that $\hat{\omega}\left(u=0, x^{A}\right)=1$. At later times, the conformal factor is calculated from the angular derivatives of the new angular coordinates via Eq. (32). The differentiation of the formulas (33) to obtain $\hat{Q}$ and $\hat{H}$ in the new coordinates is nontrivial and critical for computational steps of the regularity-preserving scheme described in Sec. V, so we give those pieces explicitly in Appendix B. The reader may refer to the metric forms (27) and (4) for reassembling the coordinate components of the metric.

At this point, the intermediate metric after step 2 already has some attractive properties. The metric is again Bondilike, and the new $\hat{U}^{A}$ vanishes at $\mathcal{I}^{+}$, which offers a number of simplifications to other metric components via the
Einstein equations. Once we have determined the corresponding $\hat{U}^{\hat{A}}, \hat{h}^{\hat{A} \hat{B}}, \hat{\beta}$, and $\hat{W}$, we may also make use of the existing formalism for evolving the Bondi-like system in the bulk. This coordinate system forms the foundation of a regularity-preserving evolution, in which the volume Cauchy-characteristic evolution is conducted in the coordinates $\left\{\hat{u}, \hat{l}, \hat{x}^{\hat{A}}\right\}$, and the world tube quantities are transformed to these coordinates before integration. In this coordinate system, if $\hat{h}^{(0) \hat{A} \hat{B}}(u=0)=q^{A B}$, then $\hat{h}^{(0) \hat{A} \hat{B}}=$ $q^{A B}$ for the entire evolution, where $\hat{h}^{(0) \hat{A} \hat{B}}$ is the asymptotic part of $\hat{h}^{\hat{A} \hat{B}}$ in the same way that $U^{(0) A}$ was defined by Eq. (29). In terms of the spin-weighted quantity $\hat{J}$, this is equivalent to saying that if $\hat{J}$ vanishes at $\mathcal{I}^{+}$on the initial data hypersurface, it will vanish at $\mathcal{I}^{+}$for the entire evolution. We call this set of intermediate coordinates the partially flat coordinates.

However, the discussion so far has not yet arrived at an asymptotically flat metric, so we return to our transformation sequence with step 3, which introduces the time coordinate $\stackrel{\circ}{u}$ such that

$$
\stackrel{\circ}{u}=\stackrel{\circ}{u^{(0)}}\left(\hat{u}, \hat{x}^{\hat{A}}\right)+\hat{l}^{\circ}(R)\left(\hat{u}, \hat{l}, \hat{x}^{\hat{A}}\right)=\int^{\hat{u}} e^{2 \hat{\beta}}+\hat{l}^{\circ} u^{(R)}\left(\hat{u}, \hat{l}, \hat{x}^{\hat{A}}\right) .
$$

We fix $\stackrel{\circ}{u^{(R)}}$ by insisting that the resulting metric has vanishing $g^{\circ \text { u } u \text {, }}$

$$
\begin{aligned}
0= & g^{\circ \stackrel{\circ}{u}}=2\left(e^{2 \hat{\beta}}+\hat{l} \partial_{\hat{u}}{ }^{\circ}{ }^{(R)}\right) \partial_{\hat{l}}\left(\hat{l}^{\circ} u^{(R)}\right) \hat{l}^{2} e^{-2 \hat{\beta}}+\hat{l}^{3} \partial_{\hat{l}}\left(\hat{l}^{\circ} u^{(R)}\right)^{2}(\hat{W}+\hat{l}) e^{-2 \hat{\beta}} \\
& +2 \hat{l}^{2}\left(\partial_{\hat{A}}{ }^{\circ}{ }^{(0)}+\hat{l} \partial_{\hat{A}}{ }^{\circ}{ }^{(R)}\right) \partial_{\hat{l}}\left(\hat{l}^{\circ} u^{(R)}\right) \hat{U}^{\hat{A}} e^{-2 \hat{\beta}}+\hat{l}^{2}\left(\partial_{\hat{A}}{ }^{\circ(0)}+\hat{l} \partial_{\hat{A}}{ }^{\circ}{ }^{(R)}\right)\left(\partial_{\hat{B}}{ }^{\circ}{ }^{(0)}+\hat{l} \partial_{\hat{B}} u^{(R)}\right) \hat{h}^{\hat{A} \hat{B}},
\end{aligned}
$$

which specifies $u^{(R)}$ as the solution to an elliptic equation. We note two possible strategies for solving (36). First, the expansion of $u^{(R)}$ in ascending powers of $\hat{l}$ gives rise to algebraic solutions for each successive expansion coefficient. For illustration, we explicitly compute the first two orders of $\stackrel{\circ}{u}^{(R)}=\stackrel{\circ}{u}^{(1)}+\hat{l}^{\circ} u^{(2)}+\mathcal{O}\left(\hat{l}^{2}\right)$ in Appendix C. Fortunately, for the discussion of asymptotic quantities in this paper, this power series solution is all that is necessary. Second, it may be possible to solve Eq. (36) for $\partial_{\hat{l}}\left(\hat{l} u^{(R)}\right)$ and numerically step $u^{(R)}$ along the hypersurface given some single sphere of values for $u^{(R)}$, though we make no claims here of the stability of such a numerical scheme.
After step 3 we are again left with a metric that is not Bondi-like, because of nonvanishing $g^{u \hat{A}}$. In the transformation step 4 , we make a subleading in $\hat{l}$ change to the angular coordinates, affecting only the values of ${ }^{\circ} \AA^{\circ}$ away from $\mathcal{I}^{+}$. The new angular coordinates take the form

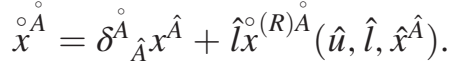

We constrain $\stackrel{\circ}{x}$ in much the same way we constrained $\stackrel{\circ}{u}$ : We impose $g^{\circ \stackrel{\circ}{A}}=0$ to preserve the Bondi form. This condition gives the equation

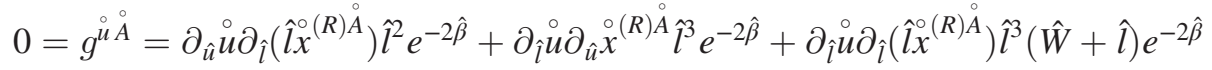

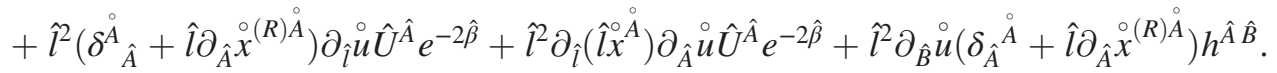


For small $\hat{l}$, Eq. (38) may be perturbatively expanded to obtain an algebraic equation for each order of $\hat{x}^{(R) \hat{A}}$, and otherwise must be numerically integrated along $\hat{l}$. The first two orders of the asymptotic expansion are provided in Appendix C.

The final coordinate transformation step 5 is closely analogous to step 2, except now we are restoring the areal radius associated with the angular coordinates $\stackrel{\circ}{x}^{A}$. Therefore, the Bondi-Sachs radial coordinate is

$$
\begin{aligned}
\stackrel{\circ}{l} & \left.=\hat{l} \sqrt{\operatorname{det}\left(\partial_{\hat{A}} \circ^{\circ} \dot{A}\right.}\right)\left(\frac{\operatorname{det}\left({\stackrel{\circ}{q_{A B} \circ}}\right)}{\operatorname{det}\left(q_{\hat{A} \hat{B}}\right)}\right)^{1 / 4} \\
& \equiv \frac{\hat{l}}{\stackrel{\circ}{\omega}\left(\hat{u}, \hat{l}, \hat{x}^{\hat{A}}\right)} \equiv \hat{l}-\hat{l}^{2} \stackrel{\circ}{(1)}^{(1)}+\mathcal{O}\left(\hat{l}^{3}\right) .
\end{aligned}
$$

In a manner analogous to Eqs. (31) and (32), $\stackrel{\circ}{\omega}$ can be written in terms of the derivatives $\partial_{A} \hat{x}^{\hat{A}}$. These derivatives are determined by solving Eq. (38) perturbatively, and doing so yields a perturbative solution for $\stackrel{\circ}{\omega}$. The expression for $\stackrel{\circ}{\omega}^{(1)}$ is provided in Appendix C.

The final formulas for the Bondi-Sachs spin-weighted scalars in the spacetime volume after all of these coordinate transformations are comparatively uninformative. Because the subleading transformations in steps 3-5 depend on all of the partially flat coordinates, all four partially flat spinweighted scalars contribute to each of the Bondi-Sachs scalars according to the standard metric transformation rules. However, the final transformations do provide compact results under perturbative expansion near $\mathcal{I}^{+}$:

$$
\begin{aligned}
& \stackrel{\circ}{\beta}^{(0)}=\stackrel{\circ}{\beta}^{(1)}=\stackrel{\circ}{J}^{(0)}=0, \\
& \stackrel{\circ}{U}^{(0)}=\stackrel{\circ}{U}^{(1)}=\stackrel{\circ}{W}^{(0)}=\stackrel{\circ}{W}^{(1)}=0 \text {, } \\
& \stackrel{\circ}{J}=\hat{J}^{(1)}+\hat{\delta}^{\circ} u^{(0)} \text {. }
\end{aligned}
$$

In some of these equations, we have further simplified using results from the perturbative expansion of the Einstein field equations.

\section{B. Inference of the news in arbitrary coordinate systems}

The Bondi news is a gauge invariant measure of the gravitational wave signal: the $\sim 1 / r$ part of the angular Bondi-Sachs metric is uniquely specified by the BondiSachs metric and falloff properties. Because this gives a unique gauge specification (up to BMS freedom), the gauge-invariant Bondi-Sachs news is defined as taking the value in every gauge that is given by transforming to the Bondi-Sachs gauge and evaluating

$$
N=\lim _{r \rightarrow \infty} \frac{1}{2 r} \stackrel{\circ}{\circ} \stackrel{\circ}{q} \stackrel{\circ}{\bar{q}}{ }^{\circ} \partial_{u} \stackrel{\circ}{h}_{A B} .
$$

Therefore, the explicit coordinate transformation given in Sec. III A gives a novel route to a gauge-invariant specification of the Bondi-Sachs news in terms of the components of a Bondi-like metric (4).

For our recommended numerical technique of evolving the characteristic system of equations in the partially flat coordinates, we determine the gauge invariant Bondi-Sachs news by expanding (41) in terms of partially flat quantities via expressions from stages 3-5 of the transformations in Sec. III A. The Bondi-Sachs news in terms of quantities in the partially flat gauge is

$$
N=e^{-2 \hat{\beta}^{(0)}}\left(\hat{\bar{H}}^{(1)}+\hat{\bar{\partial}} \hat{\bar{\partial}} e^{2 \hat{\beta}^{(0)}}\right) .
$$

In addition, we derive an equation for the Bondi-Sachs news in terms of spin-weighted scalars in an arbitrary Bondi-like gauge, which may be of use to computations that use strategies other than our partially flat coordinate system. This definition of the news is equivalent to previous expansions of the same quantity [21], but our set of explicit coordinate transformations yields simpler expressions than previously derived formulas. Our simplified expression is obtained by using the relationships between the derivatives $\hat{\partial}$ and the Bondi-like $\partial$, and the transformation of $\hat{H}$ (B3) and $\hat{\beta}$ (33a), and takes the form

$$
\begin{aligned}
& N=\frac{\hat{\omega}^{2} e^{-2 \beta^{(0)}}}{4}\left\{2\left[\hat{\bar{b}} ð \bar{U}^{(0)} J^{(1)}+\hat{a} ð U^{(0)} \bar{J}^{(1)}+\left(\hat{\bar{b}} ð U^{(0)}+\hat{a} ð \bar{U}^{(0)}\right) \operatorname{Re}\left(J^{(0)} \bar{J}^{(1)}\right)\right]\right. \\
& +\left[\hat{\bar{b}}^{2} H^{(1)}+\hat{a}^{2} \bar{H}^{(1)}+\hat{\bar{b}} \hat{a}\left(2 \operatorname{Re}\left(\boldsymbol{J}^{(0)} \bar{H}^{(1)}+\boldsymbol{J}^{(1)} \bar{H}^{(0)}\right)-\operatorname{Re}\left(\boldsymbol{J}^{(0)} \bar{H}^{(0)}\right) \operatorname{Re}\left(\boldsymbol{J}^{(0)} \bar{J}^{(1)}\right)\right)\right] \\
& +\frac{1}{2}\left[\hat{\bar{b}}^{2}\left(U^{(0)} \bar{\jmath}+\bar{U}^{(0)} \text { ð)} J^{(1)}+\hat{a}^{2}\left(U^{(0)} \overline{\hat{\jmath}}+\bar{U}^{(0)} ð\right) \bar{J}^{(1)}+\hat{a} \hat{\bar{b}}\left(U^{(0)} \bar{\jmath}+\bar{U}^{(0)} ð\right) \operatorname{Re}\left(J^{(0)} \bar{J}^{(1)}\right)\right]\right. \\
& \left.+3 \hat{\omega}^{2} \partial_{u} \hat{\omega}\left[\hat{\bar{b}} J^{(1)}+\hat{a}^{2} \bar{J}^{(1)}+\hat{a} \hat{\bar{b}} \operatorname{Re}\left(J^{(0)} \bar{J}^{(1)}\right)\right]\right\}+\frac{\hat{\omega} e^{-2 \beta^{(0)}}}{4}\left(\hat{b}^{2} ð^{2}+\hat{\bar{a}}^{2} \bar{\delta}^{2}+2 \hat{b} b \hat{\bar{a}} \bar{\varnothing}\right)\left(\frac{e^{2 \beta^{(0)}}}{\hat{\omega}}\right) .
\end{aligned}
$$




\section{COMPACTIFIED CHARACTERISTIC EVOLUTION EQUATIONS}

In this section, we present the full set of nonlinear characteristic equations using a compactified radial coordinate. We start with any coordinate system $\{u, r, \theta, \phi\}$ where the metric is in Bondi-like form, Eq. (4), and obeys the Bondi-like restrictions, Eqs (5). Note that this includes more restricted coordinates such as the partially flat coordinates of Sec. III A and true Bondi-Sachs coordinates, so the results of this section are applicable to those more restricted coordinates as well.

The characteristic equations presented here are equivalent to a coordinate-transformed version of the equations derived by [21] but take a newly updated form. The new compactified radial coordinate gives the left-hand sides of the differential equations a standard and convenient form, and because it is defined on $[-1,1]$ it facilitates standard spectral tools such as Legendre or Chebyshev polynomial representations. In addition to introducing a compactified coordinate, we have also manipulated the equations in the following ways to better suit numerical implementation:

(1) All spin-weighted derivatives result in a spin weight between 2 and -2 , so that spin-weighted transform libraries with a hard limit at spin weight 2 can be used.

(2) All derivatives of $K$ are expanded and, to the extent possible, simplified with other terms involving $J$.

(3) Where relevant, terms that nearly cancel have been reduced to expressions of similar numerical magnitude. For example, consider the term $1-K$. If evaluated directly for small $J$, there will be loss of numerical precision. In the extreme case where $J$ is on the order of the square root of machine epsilon, then $K$ will be unity to machine precision and $1-K$ will be entirely error. Our solution is to evaluate $1-K$ as $\left(1-K^{2}\right) /(1+K)=J \bar{J} /(1+K)$, which retains full precision even when $J$ is small.

(4) For applicable equations, many collections of terms that appear along with their complex conjugates are identified so that several numerical steps can be avoided by caching a quantity and negating its imaginary part.

Given any Bondi-like (including even more restricted) coordinates $\{u, r, \theta, \phi\}$, we define compactified coordinates $\{\breve{u}, \breve{y}, \breve{\theta}, \breve{\phi}\}$. The numerical coordinates are identical to the Bondi-like coordinates aside from the radial component,

$\breve{u}=u, \quad \breve{y}=1-\frac{2 R}{r}, \quad \breve{\theta}=\theta, \quad \breve{\phi}=\phi$,

where $R$ is the Bondi-like radius of the world tube $\Gamma$,

$$
R(u, \theta, \phi)=\left.r\right|_{\Gamma}
$$

By construction, $\Gamma$ is a surface of constant $y=-1$, but not a surface of constant Bondi-like radius $r$.

Inverting the Jacobian gives the necessary factors to convert between the Bondi-like derivatives and the derivatives with respect to the numerical coordinates:

$$
\begin{aligned}
\partial_{r} & =\frac{(1-\breve{y})^{2}}{2 R} \partial_{\breve{y}}, \\
\partial_{u} & =\partial_{\breve{u}}-(1-\breve{y}) \frac{\partial_{\breve{u}} R}{R} \partial_{\breve{y}}, \\
ð & =\breve{ð}-(1-\breve{y}) \frac{\breve{\partial} R}{R} \partial_{\breve{y}} .
\end{aligned}
$$

In the following discussion, there is often little to be gained from explicitly converting the angular derivatives $\partial$

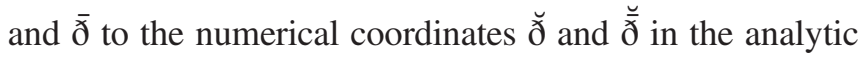
equations. In virtually every case, the numerical angular derivatives do not simplify the expression. Further, new terms introduced by the angular Jacobians are better behaved at $\mathcal{I}^{+}$, so the statements about the pole structure of the equations carry through without modification. In cases where $ð$ and $ð$ act on functions of $\breve{x^{\mu}}$, the needed Jacobian factors from (46) are implied. For a practical implementation, the appropriate Jacobian factors should be included in the subroutines that calculate the angular derivatives $\partial$ and $\bar{\partial}$. However, it is valuable to convert explicit factors of $r$ and derivatives with respect to $r$ to numerical coordinates, as such factors can alter the pole structure in the numerical coordinates and offer subtle simplifications.

The results of this section are the new explicit forms of the equations themselves and so are presented in full in Secs. IV B-IV F with minimal further exposition on their structure. The reader is invited to download the Mathematica companion notebook to this paper [39], which provides tools for confirming the compactified characteristic equations and their digital forms for further exploration. The equations are given in terms of Bondi-like spin-weighted scalars $\breve{J}, \breve{\beta}, \breve{Q}, \breve{U}, \breve{W}$, and $\breve{H}$ in the compactified coordinates. Most of these scalars depend on the coordinate derivatives only through the equations of motion, so the quantities act as scalars for the transformation $\breve{F}=F$, for all $F \in\{J, \beta, Q, U, W\}$, and

$$
\breve{H}=H+\partial_{u} R \partial_{r} J .
$$

\section{A. Common forms of hypersurface equations}

Each of the hypersurface equations (23) can be placed into one of three categories, depending on the pole structure of the equation [arising from terms such as $(1-\breve{y})^{n}$ that vanish at $\mathcal{I}^{+}$] and the form of the terms that involve the variable that is determined by that equation. We discuss these categories in terms of our compactified radial 
coordinate $\breve{y}$, but most statements here would be similarly applicable to any choice of radial coordinate. The first and simplest type is those hypersurface equations that govern the quantities $\beta$ and $U$. These have the form

$$
\partial_{\breve{y}} F_{1}\left(\breve{x^{\breve{\mu}}}\right)=S_{1}\left(\breve{x^{\mu}}\right)
$$

where $F_{1}$ is the quantity determined by the equation and $S_{1}$ is a nonlinear expression that is independent of $F_{1}$ but may involve other already-known hypersurface quantities. For this category, the integral determining $F_{1}$ can be evaluated in a straightforward way by standard ODE methods.

The second category contains the hypersurface equations that govern the computation of $Q$ and $W$. These possess the form

$$
\begin{aligned}
& (1-\breve{y}) \partial_{\breve{y}} F_{2}\left(\breve{x}^{\breve{\mu}}\right)+2 F_{2}\left(\breve{x}^{\breve{\mu}}\right) \\
& \quad=S_{2}^{P}\left(\breve{x}^{\breve{\mu}}\right)+(1-\breve{y}) S_{2}^{R}\left(\breve{x}^{\breve{\mu}}\right) .
\end{aligned}
$$

Here $F_{2}$ is the quantity being solved for, and $S_{2}^{P}$ and $S_{2}^{R}$ are known nonlinear expressions independent of $F_{2}$. This form of equation requires significantly more care than Eq. (48) because of the $(1-\breve{y})$ terms. Depending on the method of solution, erroneous logarithm dependence may arise from manipulation of the differential equation [31]. Further, the known functions $S_{2}^{P}$ and $S_{2}^{R}$ on the right-hand side of Eq. (49) produce a quadratic contribution when they are expanded as a power series in $(1-\breve{y})$ near $\breve{y}=1$. Unless there is an explicit cancellation of this quadratic in the $(1-\breve{y})$ contribution, this equation produces pure-gauge logarithm dependence in the solution to $F_{2}$. In Sec. III we present a set of coordinate transformations that we use in Sec. V to construct a complete method for avoiding the logarithm dependence in these contributions to the Bondilike characteristic equations.

The third and final form of the differential equation found among the characteristic hypersurface equations governs only the quantity $H$. It takes the form

$$
(1-\breve{y}) \partial_{\breve{y}} F_{3}\left(\breve{x}^{\breve{\mu}}\right)+\left[1+(1-\breve{y}) L_{3}^{G}\left(\breve{x}^{\breve{\mu}}\right) L_{3}^{J}\left(\breve{x}^{\breve{\mu}}\right)\right] F_{3}\left(\breve{x}^{\breve{\mu}}\right)+(1-\breve{y}) \bar{L}_{3}^{G}\left(\breve{x}^{\breve{\mu}}\right) L_{3}^{J}\left(\breve{x}^{\breve{\mu}}\right) \bar{F}_{3}\left(\breve{x}^{\breve{\mu}}\right)=S_{3}^{P}\left(\breve{x}^{\breve{\mu}}\right)+(1-\breve{y}) S_{3}^{R}\left(\breve{x}^{\breve{\mu}}\right) .
$$

Here $F_{3}$ is the quantity being solved for, and $S_{3}^{P}, S_{3}^{R}, L_{3}^{G}$, and $L_{3}^{J}$ are known nonlinear expressions independent of $F_{3}$. The treatment of the poles for this equation is similar to that used for Eq. (49), except that it is now the linear in $(1-\breve{y})$ term that governs the logarithmic dependence. The form (50) has the further complication of the nonderivative term depending both on $F_{3}$ and its complex conjugate $\bar{F}_{3}$, which prevents a simple single-pass integration in spectral representation that is possible for (48) and (49). Instead, we decompose the equation and the individual factors into real and imaginary parts. The matrix form of Eq. (50) is [28]

$$
\begin{aligned}
& \left(\left[\begin{array}{cc}
(1-y) \partial_{y}+1 & 0 \\
0 & (1-y) \partial_{y}+1
\end{array}\right]+(1-y)\left[\begin{array}{cc}
\operatorname{Re}\left(L_{3}^{J}\right) \operatorname{Re}\left(L_{3}^{G}\right) & \operatorname{Re}\left(L_{3}^{J}\right) \operatorname{Im}\left(L_{3}^{G}\right) \\
\operatorname{Im}\left(L_{3}^{J}\right) \operatorname{Re}\left(L_{3}^{G}\right) & \operatorname{Im}\left(L_{3}^{J}\right) \operatorname{Im}\left(L_{3}^{G}\right)
\end{array}\right]\right)\left[\begin{array}{l}
\operatorname{Re}\left(L_{3}\right) \\
\operatorname{Im}\left(L_{3}\right)
\end{array}\right] \\
& \quad=\left[\begin{array}{l}
\operatorname{Re}\left(S_{3}^{P}\right)+(1-y) \operatorname{Re}\left(S_{3}^{R}\right) \\
\operatorname{Im}\left(S_{3}^{P}\right)+(1-y) \operatorname{Im}\left(S_{3}^{R}\right)
\end{array}\right] .
\end{aligned}
$$

\section{B. Hypersurface equation: $\beta$}

The first equation to be evaluated on each hypersurface is the one that determines $\beta$ given $J$ on the same hypersurface:

$$
\partial_{r} \beta=-\frac{1}{8} r\left(\partial_{r} J \partial_{r} \bar{J}-\frac{\left(\partial_{r}(J \bar{J})\right)^{2}}{4 K^{2}}\right) .
$$

Converting to numerical coordinates yields

$$
\partial_{\breve{y}}(\breve{\beta})=-\frac{1}{8}(1-\breve{y})\left(\partial_{\breve{y}} \breve{J} \partial_{\breve{y}} \breve{J}-\frac{\left(\partial_{\breve{y}}(\breve{J} \breve{\breve{J}})\right)^{2}}{4 \breve{K}^{2}}\right) .
$$

This equation takes the form (48) and can be integrated by traditional methods.

\section{Hypersurface equation: $Q$}

The second hypersurface equation evaluated on each hypersurface determines the value of $Q$ given $J$ and $\beta$. Our simplified form of the $Q$ hypersurface equation in Bondilike coordinates is

$$
\partial_{r}\left(Q r^{2}\right)=-r^{2}\left(\Lambda_{Q}+\frac{\bar{\Lambda}_{Q} J}{K}+\frac{\partial_{r} \bar{\partial} J}{K}\right)+2 r^{4} \partial_{r}\left(\frac{\partial \beta}{r^{2}}\right),
$$

where

$$
\begin{aligned}
\Lambda_{Q}= & -\frac{1}{2} ð\left(\bar{J} \partial_{r} J\right)+\frac{1}{2} J \partial_{r} \partial \bar{J} \\
& -\frac{1}{2} \partial \bar{J} \partial_{r} J+\frac{ð(J \bar{J}) \partial_{r}(J \bar{J})}{4 K^{2}} .
\end{aligned}
$$


These equations become, when converted to compactified coordinates,

$$
2 \breve{Q}+(1-\breve{y}) \partial_{\breve{y}} \breve{Q}=-4 ð \breve{\beta}-(1-\breve{y})\left(2 \Lambda_{\breve{Q}}+\frac{2 \bar{\Lambda}_{\breve{Q}} \breve{J}}{\breve{K}}-2 ð \partial_{\breve{y} \breve{\beta}}+\frac{\bar{\partial} \partial_{\breve{y}} \breve{J}}{\breve{K}}-\frac{2 ð R \partial_{\breve{y} \breve{\beta}} \breve{\partial} R \partial_{\breve{y}} \breve{J}}{R}\right)
$$

where we have introduced

$$
\Lambda_{\breve{Q}}=-\frac{1}{4} ð\left(\breve{\breve{J}} \partial_{\breve{y}} \breve{J}\right)+\frac{1}{4} \breve{J} ð \partial_{\breve{y}} \breve{J}-\frac{1}{4} ð \breve{J} \partial_{\breve{y}} \breve{J}+\frac{ð(\breve{J} \breve{J}) \partial_{\breve{y}}(\breve{J} \breve{J})}{8 K^{2}}+\frac{\partial^{\prime}(R)\left(\breve{J} \partial_{\breve{y}}^{\breve{J}}-\breve{J} \partial_{\breve{y}} \breve{J}\right)}{4 R} .
$$

The pole structure is easily inferred from the form of Eq. (56). We identify the first and second terms of the righthand side with $S_{\breve{Q}}^{P}$ and $S_{\breve{Q}}^{R}$ from (49).

\section{Hypersurface equation: $U$}

The next equation in the sequence determines the value of $U$ (analogous to an angular shift contribution in the $\mathrm{ADM}$ formalism) on a given hypersurface given the values of $J, \beta$, and $Q$. In Bondi-like coordinates

$$
\partial_{r} U=\frac{e^{2 \beta}}{r^{2}}(K Q-J \bar{Q}) .
$$

The $U$ equation is of unusual simplicity for the characteristic formulation, so the conversion to our adjusted compactified form is straightforward:

$$
\partial_{\breve{y}} \breve{U}=\frac{e^{2 \breve{\beta}}}{2 R}(\breve{K} \breve{Q}-\breve{J} \breve{\bar{Q}}) .
$$

Similar to the equation for $\breve{\beta}$, the hypersurface equation for $\breve{U}$ is of the form (48) and can be integrated by traditional means.

\section{E. Hypersurface equation: $W$}

The fourth equation in the hypersurface integration sequence determines the value of the "mass aspect" contribution $W$ on a $u=$ const hypersurface given values of $J, \beta, Q$, and $U$ :

$$
\begin{aligned}
\partial_{r}\left(r^{2} W\right)= & 1+\frac{1}{2} e^{2 \beta}\left(\Lambda_{W}+\Lambda_{W}\right)+(ð \bar{U}+\bar{ð} U) r \\
& +\frac{1}{4}\left(\partial_{r} \partial \bar{U}+\partial_{r} \bar{\partial} U\right) r^{2},
\end{aligned}
$$

where

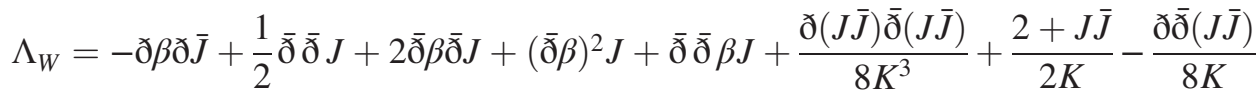

$$
\begin{aligned}
& -\frac{\partial(J \bar{J}) \bar{\partial} \beta}{2 K}-\frac{\partial \bar{J} \bar{\partial} J}{4 K}-\frac{\bar{J} ð \bar{\partial} J}{4 K}-K ð \bar{ð} \beta-K ð \beta \bar{\delta} \beta+\frac{1}{4}\left(-K Q \bar{Q}+J \bar{Q}^{2}\right) .
\end{aligned}
$$

The conversion to numerical coordinates for this equation proceeds simply, as no term in $\Lambda_{W}$ requires any alteration (replace $\beta, J, K$, and $Q$ with $\breve{\beta}, \breve{J}, \breve{K}$, and $\breve{Q}$ ). We rearrange to make explicit the pole structure of the equation,

$$
2 \breve{W}+(1-\breve{y}) \partial_{\breve{y}} \breve{W}=(ð \breve{U}+\bar{\jmath} \breve{U})+(1-\breve{y})\left(\frac{1}{4} ð \partial_{\breve{y}} \breve{U}+\frac{1}{4} \bar{\partial} \partial_{\breve{y}} \breve{U}+\frac{1}{4} \partial_{\breve{y}} \breve{U} \frac{\bar{\partial} R}{R}+\frac{1}{4} \partial_{\breve{y}} \breve{U} \frac{\partial R}{R}-\frac{1}{2 R}+\frac{e^{2 \breve{\beta}}\left(\Lambda_{\breve{W}}+\bar{\Lambda}_{\breve{W}}\right)}{4 R}\right) .
$$

Again drawing the comparison to (49), we identify the first and second terms on the right-hand side of (62) as $S_{\breve{W}}^{P}$ and $S_{\breve{W}}^{R}$.

\section{F. Hypersurface equation: $\boldsymbol{H}$}

The final hypersurface equation determines the value of $\partial_{u} J \equiv H$, which is the sole time-derivative quantity used in the characteristic evolution computation. Once determined by radial integration, the numerical time derivative $\partial_{\breve{u}} J$ is used in a standard numerical ODE integrator (e.g., RungeKutta or adaptive Dormand-Prince) to determine the value of $J$ on subsequent hypersurfaces. This value is then used as the input for the hypersurface computations of the metric components on subsequent hypersurfaces.

The simplified hypersurface equation for $H$ in Bondilike coordinates is 


$$
\begin{aligned}
\partial_{r}( & H r)+J r\left(\mathcal{L}_{H} H+\overline{\mathcal{L}}_{H} H\right) \\
= & -\frac{1}{2} ð\left(\left(J+r \partial_{r} J\right) \bar{U}\right)+\left(\mathcal{B}_{H}+\overline{\mathcal{B}}_{H}\right) J-J\left(ð \bar{U}+\frac{1}{2} \bar{ð} U\right)-K ð U-\frac{1}{2} \partial_{r}(r \bar{ð} J) U+\frac{1}{2} \partial_{r} \partial_{r} J\left(r^{2} W+r\right) \\
& +\frac{e^{2 \beta}}{r}\left[\frac{1}{2} \bar{ð} J K \mathcal{C}_{H}+\frac{\overline{\mathcal{C}}_{H} J^{2} \partial \bar{J}}{2 K}-\left(\mathcal{A}_{H}+\overline{\mathcal{A}_{H}}+\frac{\partial \overline{\mathcal{C}}_{H}}{2 K}\right) J+ð C_{H}-\frac{\partial\left(J \overline{\mathcal{C}}_{H}\right)}{2 K}+\mathcal{C}_{H}^{2}\right] \\
& +\partial_{r}(J)\left[1+\frac{1}{2} r^{2} \partial_{r} W+\frac{3}{2} r W-\frac{1}{2}\left(-\bar{\partial} \bar{U} J+\frac{\partial U \bar{J}}{K^{2}}\right) K r-\frac{1}{4} D_{H} K^{2} r\right] \\
& +\partial_{r}(\bar{J}) J^{2} r\left[\frac{1}{4} D_{H}+\frac{1}{2 K} ð U \bar{J}\right],
\end{aligned}
$$

where

$$
\begin{aligned}
& \mathcal{A}_{H}=\frac{1}{4} ð(ð(\bar{J}))-\frac{\partial \bar{\partial}(J \bar{J})-2 \bar{J} ð \bar{\partial} J}{16 K^{3}}-\frac{\bar{J} ð \bar{\partial} J-3}{4 K}+\frac{1}{2} ð(\bar{J})\left(C_{H}+\frac{\bar{\partial}(J \bar{J}) J}{4 K^{3}}-\frac{\bar{\partial} J(2 J \bar{J}+1)}{4 K^{3}}\right), \\
& \mathcal{B}_{H}=\frac{1}{2 r}+W+\frac{1}{2} r \partial_{r} W+2 \partial_{r} \beta(r W+1) \\
& -\frac{\partial U \bar{J} \partial_{r}(J \bar{J}) r}{4 K}+\frac{r U}{4}\left(\bar{\partial} J \partial_{r} \bar{J}+\bar{ð}\left(\bar{J} \partial_{r} J\right)-\bar{J} \partial_{r} \bar{\partial} J-\frac{\bar{\delta}(J \bar{J}) \partial_{r}(J \bar{J})}{2 K^{2}}\right), \\
& \mathcal{C}_{H}=ð \beta-\frac{1}{2} Q, \\
& \mathcal{D}_{H}=ð \bar{U}-\bar{ð} U, \\
& \mathcal{L}_{H}=\frac{1}{2}\left(-\partial_{r} \bar{J}+\frac{\bar{J} \partial_{r}(J \bar{J})}{2 K^{2}}\right) .
\end{aligned}
$$

The equation for $H$ is considerably more intricate than the other hypersurface equations. When writing it in numerical coordinates, note that we must also rewrite the evolution equation $\partial_{u} J=H$ as $\partial_{\breve{u}} \breve{J}=\breve{H}$, where $\breve{H}$ is related to $H$ by Eq. (47). The hypersurface equation for $\breve{H}$ takes the form (50)

$$
(1-\breve{y}) \partial_{\breve{y}} \breve{H}+\breve{H}+(1-\breve{y}) \breve{J}\left(\mathcal{L}_{\breve{H}} \breve{H}+\overline{\mathcal{L}}_{\breve{H}} \breve{\bar{H}}\right)=S_{\breve{H}}^{P}+(1-\breve{y}) S_{\breve{H}}^{R}
$$

with source terms

$$
\begin{aligned}
& \mathcal{L}_{\breve{H}}=\frac{1}{2}\left(-\partial_{\breve{y}} \breve{\bar{J}}+\frac{\breve{\bar{J}} \partial_{\breve{y}}(\breve{J} \breve{\bar{J}})}{2 \breve{K}^{2}}\right), \\
& S_{\breve{H}}^{P}=-\frac{1}{2}(ð(\breve{J} \breve{U})+\bar{ð}(\breve{J} \breve{U})+2 J ð \bar{U})+2 \breve{W} \breve{J}-\breve{K} ð \breve{U}, \\
& S_{\breve{H}}^{R}=\breve{J}\left(\mathcal{B}_{\breve{H}}+\overline{\mathcal{B}}_{\breve{H}}\right)+\frac{1}{2}(1-\breve{y})\left(\breve{W}+\frac{(1-\breve{y})}{2 R}+2 \frac{\partial_{\breve{u}} R}{R}\right) \partial_{\breve{y}}^{2} \breve{J}-\frac{1}{2}\left(\breve{U} \bar{\partial} \partial_{\breve{y}} \breve{J}+\breve{\breve{U}} \partial_{\breve{y}} \breve{J}\right)+\breve{J}^{2} \partial_{\breve{y}} \breve{\breve{J}}\left[\frac{\mathcal{D}_{\breve{H}}}{4}+\frac{\partial \breve{U} \breve{J}}{2 \breve{K}}\right]
\end{aligned}
$$

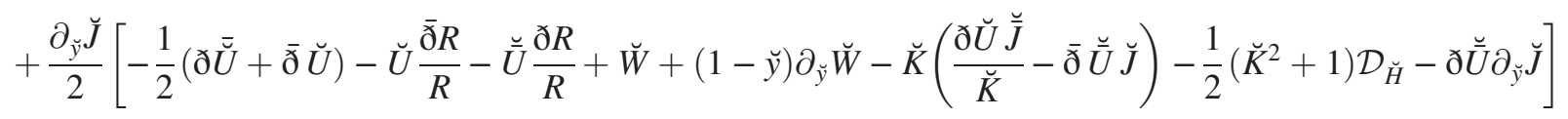

$$
\begin{aligned}
& +\frac{e^{2 \breve{\beta}}}{2 R}\left[\frac{J}{2 K^{3}}+\frac{1}{2} \bar{\partial} \breve{J} \breve{K} \mathcal{C}_{\breve{H}}+\frac{\overline{\mathcal{C}}_{\breve{H}} \breve{J}^{2} ð \breve{J}}{2 \breve{K}}-\left(\mathcal{A}_{\breve{H}}+\overline{\mathcal{A}}_{\breve{H}}\right) \breve{J}+ð C_{\breve{H}}-\frac{\overline{\mathcal{C}}_{\breve{H}} ð \breve{J}}{2 \breve{K}}+\mathcal{C}_{\breve{H}}^{2}\right]
\end{aligned}
$$

where $\mathcal{A}_{\breve{H}}, \mathcal{C}_{\breve{H}}$, and $\mathcal{D}_{\breve{H}}$ have the same form as $\mathcal{A}_{H}, \mathcal{C}_{H}$, and $\mathcal{D}_{H}$ with the replacements $\{J, \beta, Q, U, W\} \rightarrow\{\breve{J}, \breve{\beta}, \breve{Q}, \breve{U}, \breve{W}\}$. The quantity $\mathcal{B}_{\breve{H}}$ is defined as 


$$
\begin{aligned}
\mathcal{B}_{\breve{H}}=\frac{1}{2}\left[\frac{1}{2 R}+\partial_{\breve{y}} \breve{W}+\left(\breve{W}+\frac{1-\breve{y}}{2 R}+2 \frac{\partial_{\breve{u}} R}{R}\right) \partial_{\breve{y}} \breve{\beta}-\frac{\partial \breve{U} \breve{\breve{J}} \partial_{\breve{y}}(\breve{J} \breve{\bar{J}})}{2 \breve{K}}\right. \\
\left.+\frac{\breve{U}}{2}\left(\bar{ð} \breve{J} \partial_{\breve{y}} \breve{J}+\bar{\jmath}\left(\breve{\bar{J}} \partial_{\breve{y}} \breve{J}\right)-\breve{J} \bar{\partial} \partial_{\breve{y}} \breve{J}-\frac{\bar{\partial}(\breve{J} \breve{\bar{J}}) \partial_{\breve{y}}(\breve{J} \breve{J})}{2 \breve{K}}\right)\right] .
\end{aligned}
$$

\section{REGULARITY-PRESERVING CCE}

One of the most notable drawbacks of previous implementations [28] of spectral CCE was the presence of puregauge logarithmic dependence, where spin-weighted scalars such as $Q, W$, and $H$ developed behavior like $r^{-n} \ln (r)$ at large $r$. The logarithmic dependence is particularly troubling for spectral treatments of the system, which rely on the representability of all solutions as a rapidly converging polynomial series in the compactified radial coordinate. This problem was partially mitigated in the updated implementation [31], which ensures that no logarithmic behavior is introduced by inadequate numerical treatment of the equations if such behavior is not present in the true solutions of those equations. However, there is no guarantee that the true solutions of the characteristic equations lack all logarithmic dependence in an arbitrary Bondi-like gauge.

In this section, we present the necessary conditions for the CCE system to remain regular (all quantities are polynomial in $r^{-1}$ ) at $\mathcal{I}^{+}$as the system is evolved. We demonstrate that the intermediate gauge, referred to here as the partially flat gauge, is sufficient to guarantee asymptotically well-behaved spin-weighted scalars throughout the evolution. We then synthesize the collection of our suggested improvements in a complete description of an improved Cauchy-characteristic evolution algorithm in Sec. V C.

\section{A. An overview of the regularity conditions in abstract notation}

The hypersurface equations for $Q, W$, and $H$ have nontrivial pole structures. In this section, we explore the pole structures and the consequences of the partially flat gauge developed in Sec. III for the regularity of $\hat{Q}, \hat{W}$, and $\hat{H}$. To avoid complications with order counting for powers of $r$ when derivatives are involved, we adopt the frequently used notation $l \equiv r^{-1}$ to describe asymptotic dependence, where $l=0$ at $\mathcal{I}^{+}$. Note also that the reasoning presented in this section applies similarly to the numerical coordinates, using $\breve{l}=(1-\breve{y})$. For simplicity of notation, we present the regularity conditions in the Bondi-like coordinates, and note that if the spin-weighted scalars are regular in Bondilike $l$ at $\mathcal{I}^{+}$, they are also regular in the associated $(1-\breve{y})$ numerical coordinate.

The $Q$ and $W$ equations take the form (49). Expressed in terms of $l$, we have the form

$$
\partial_{l}\left(\frac{F_{2}}{l^{2}}\right)=\frac{S_{2}^{P}}{l^{3}}+\frac{S_{2}^{R}}{l^{2}} .
$$

For generic right-hand side factors $S_{2}^{P}$ and $S_{2}^{R}$, which depend on $l$, there is the danger that the right-hand side asymptotically scales as $l^{-1}$. If this happens, either initially or during an evolution, the solution for $Q$ or $W$ will behave asymptotically as $l^{2} \ln (l)$. The conditions to avoid such logarithmically dependent terms in these equations are the regularity conditions on the hypersurface sources for the characteristic equations. For the above equation (68), the regularity condition is

$$
\left.\frac{1}{2} \partial_{l}^{2} S_{2}^{P}\right|_{l=0}=-\left.\partial_{l} S_{2}^{R}\right|_{l=0} .
$$

Similar conditions apply to the hypersurface equation that determines the evolution quantity $\partial_{u} J \equiv H$ [cf. Eq. (50)], which we write in terms of $l$ as

$$
\partial_{l}\left(\frac{F_{3}}{l}\right)+\frac{1}{l}\left(L_{F_{3}} F_{3}+L_{\bar{F}_{3}} \bar{F}_{3}\right)=\frac{S_{3}^{P}}{l^{2}}+\frac{S_{3}^{R}}{l} .
$$

The regularity condition for Eq. (70) is

$$
\left.\partial_{l} S_{3}^{P}\right|_{l=0}=-\left.S_{3}^{R}\right|_{l=0} .
$$

Note that because $H=\partial_{u} J, H$ must also obey any requirement imposed on $J$ by the regularity conditions (69) and (71); otherwise, $J$ will fail to obey the regularity requirements as it evolves forward in time.

\section{B. Explicit form of the regularity conditions}

Here, we present the conclusions regarding the regularity of the set of hypersurface and evolution equations obtained by perturbatively expanding each equation near $\mathcal{I}^{+}$. In each step, we fix the first few powers in $l$ of the spin-weighted scalar in question needed for subsequent steps, identify components informed by boundary data, and determine the constraints implied by the regularity conditions (69) and (71). In particular, we show that the partially flat gauge established in Sec. III is sufficient to ensure regularity of the hypersurface equations when supplied with appropriate initial data for $\hat{J}$. It is important to note that a partially flat gauge is not necessary, and more complicated gauge conditions can be imposed to ensure regularity in more 
generic Bondi-like gauges. In the below expansions, we formally impose the partially flat requirements $\hat{J}^{(0)}=$ $\hat{U}^{(0)}=0$. All other results follow from these requirements used in conjunction with the hypersurface equations from Sec. IV and the regularity conditions (69) and (71). In this section, we use expansion notation consistent with Sec. III; for instance, $\beta=\beta^{(0)}+l \beta^{(1)}+l^{2} \beta^{(2)}+\mathcal{O}\left(l^{3}\right)$.

The $\hat{\beta}$ hypersurface equation (52) gives rise to no regularity conditions; if $\hat{J}$ is regular at $\mathcal{I}^{+}$, then so is $\hat{\beta}$. Expanding the generic $\hat{\beta}$ hypersurface equation order by order assuming $\hat{J}$ regularity, we conclude that for a partially flat gauge,

$$
\begin{aligned}
& \hat{\beta}^{(1)}=0, \\
& \hat{\beta}^{(2)}=\frac{\hat{\bar{J}}^{(1)} \hat{J}^{(1)}}{16},
\end{aligned}
$$

and $\hat{\beta}^{(0)}$ is fixed by the boundary conditions.

The $\hat{Q}$ hypersurface equation (54) gives rise to the requirements

$$
\begin{aligned}
& \hat{Q}^{(0)}=-2 \hat{\delta} \hat{\beta}^{(0)}, \\
& \hat{Q}^{(1)}=\hat{\widehat{\partial}} \hat{J}^{(1)} .
\end{aligned}
$$

So, the $\mathcal{O}(l)$ part of the $Q$ hypersurface equation (73b) gives rise to the regularity condition

$$
\hat{J}^{(2)}=0,
$$

and the $\hat{Q}^{(2)}$ part of the expansion is determined by the boundary data on the hypersurface.

The regularity of $\hat{U}$ follows from the regularity of the previously discussed $Q, J$, and $\beta$. However, for subsequent equations, we require the results from the perturbative expansion of the $\hat{U}$ hypersurface equation (58):

$$
\begin{aligned}
& \hat{U}^{(1)}=2 e^{2 \hat{\beta}^{(0)}} \hat{\mathrm{\partial}} \hat{\beta}^{(0)}, \\
& \hat{U}^{(2)}=-\frac{e^{2 \hat{\beta}^{(0)}}\left(\hat{\bar{\partial}} \hat{J}^{(1)}+2 \hat{\bar{\delta}} \hat{\beta}^{(0)} \hat{J}^{(1)}\right)}{2} .
\end{aligned}
$$

From the expansion of the $\hat{W}$ hypersurface equation (60), we have the order-by-order constraints:

$$
\begin{aligned}
& \hat{W}^{(0)}=0,
\end{aligned}
$$

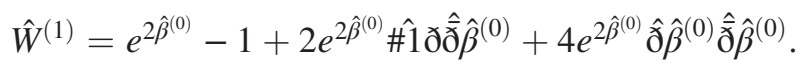

The regularity of $\hat{W}$ is directly satisfied from the previous identities, so it imposes no further conditions.

Finally, we consider Eq. (63) for $\hat{H}$. The regularity condition again demands that the $\sim l$ part of the right-hand side vanishes, which is already satisfied given the conditions of the partially flat gauge and the results (72)-(76). The expansion of $\hat{H}$ also fixes

$$
\begin{aligned}
& \partial_{\hat{u}} \hat{J}^{(0)}=0, \\
& \partial_{\hat{u}} \hat{J}^{(2)}=0,
\end{aligned}
$$

and $\partial_{\hat{u}} \hat{J}^{(1)}$ is fixed by boundary conditions. The constraints given by (77) are important, as they indicate that the previous requirements constructed to ensure regularity are stable in the evolution system.

We conclude from the full examination of the characteristic system of equations that to ensure regularity for the entire evolution, it is sufficient to impose the two conditions:

(i) Partially flat gauge;

(ii) $\hat{J}^{(2)}=0$ on the initial hypersurface.

Without these requirements, world tube data provided in an arbitrary Bondi-like gauge will tend to have small violations of the regularity conditions and produce slowly growing logarithmic terms that undermine the precision of spectral techniques.

\section{Computational gauge strategy}

In the above Secs. VA and V B, we have demonstrated that under a particular set of asymptotic flatness requirements, the characteristic evolution system remains regular and possesses desirable computational properties. However, in standard CCE implementations, the Bondi-like coordinates are subject to the arbitrary gauge provided by the world tube data, and it is not immediately obvious how we might practically implement the transformations necessary to keep the evolution system in the partially flat gauge.

In this section, we present a sketch of the computational strategy for imposing the partially flat gauge given the typical starting point of unfixed data on the initial hypersurface $u=0$ and boundary data provided in a Bondilike gauge at the world tube. The procedure we outline below demonstrates that it is possible to obtain all of the information necessary to impose regularity and partially flat gauge without disrupting the hierarchical structure of the characteristic system of Eqs. (23).

Initialization:

(i) Initialize the partially flat angular coordinates $\hat{x}^{\hat{A}}$ on the initial hypersurface $u=0$ as the Bondi-like angular coordinates $\hat{x}^{\hat{A}}(u=0)=x^{A}(u=0)$.

(ii) Initialize $\hat{J}$ by using the provided boundary value of $J$ (for the initial time the angular transformation is trivial), with a $r^{-1}$ falloff. Further terms may be 
added to adhere more tightly to the boundary data, but no term should be added that has either $r^{0}$ or $r^{-2}$ dependence near $\mathcal{I}^{+}$.

At the start of the evolution, we know $\partial_{A} \hat{x}^{\hat{A}}$ at the current $u$, but we do not know $\partial_{u} \hat{x}^{\hat{A}}$, as the time derivative requires knowledge of $U^{(0)}$ [cf. Eq. (28)].

Evolution:

(i) Compute $\left.\hat{\beta}\right|_{\Gamma}$ on the world tube using the conversion equation (33) and interpolating to the grid associated with the new angular coordinates $\hat{x}^{\hat{A}}$, and then evaluate the $\hat{\beta}$ hypersurface equation (53), taking as input $\left.\hat{\beta}\right|_{\Gamma}$ and $\hat{J}$.

(ii) Compute $\left.\hat{Q}\right|_{\Gamma}$ on the world tube using the transformation equation (B1) and interpolating to $\hat{x}^{\hat{A}}$, and then evaluate the $\hat{Q}$ hypersurface equation (56) using the boundary value $\left.\hat{Q}\right|_{\Gamma}$ and the previously determined $\hat{\beta}$ and $\hat{J}$.

(iii) The $U$ equation requires care. Note that at this point, we do not know $U^{(0)}$ at the current time step, so we must find a method of determining both $U^{(0)}$ and $\hat{U}$ given what we have so far calculated. To accomplish this, we make use of

$$
\mathcal{U}=\frac{1}{2}(\hat{\bar{b}} U-\hat{a} \bar{U})-\frac{e^{2 \hat{\beta}}}{\hat{\omega} \hat{r}}(\hat{\partial} \hat{\omega} \hat{K}-\hat{\bar{\partial}} \hat{\omega} \hat{J}) .
$$

Note that $\mathcal{U}-\hat{U}=-(1 / 2)\left(\hat{\bar{b}} U^{(0)}-\hat{a} \bar{U}^{(0)}\right)$, so $\partial_{r} U=\partial_{r} \hat{U}$, so we may use as the integrand for $\mathcal{U}$ the right-hand side of (59), taking as inputs the previously calculated Bondi quantities $\hat{J}, \hat{\beta}$, and $\hat{Q}$. Then, to integrate $\mathcal{U}$, we evaluate (78) on the boundary and integrate the right-hand side evaluated from partially flat quantities $\hat{J}, \hat{\beta}$, and $\hat{Q}$.

(iv) Determine the $I^{+}$value $U^{(0)}$ from the $\mathcal{I}^{+}$value of $\mathcal{U}$ determined in the previous step. This involves only the mild inconvenience of the complex matrix inversion, giving

$$
U^{(0)}=\frac{2}{\hat{\omega}^{2}}\left(b \mathcal{U}^{(0)}+a \bar{U}^{(0)}\right) .
$$

We have now established the rest of the coordinate transformation Jacobian

$$
\partial_{u} \hat{x}^{\hat{A}}=-U^{(0) B} \partial_{B} \hat{x}^{\hat{A}},
$$

So the remaining steps of the characteristic evolution proceed comparatively directly.

(v) Evaluate $\hat{U}=\mathcal{U}-\mathcal{U}^{(0)}$, which is the required spinweighted scalar in the partially flat gauge to be used in the remainder of the calculation.

(vi) The boundary condition for $\left.\hat{W}\right|_{\Gamma}$ is given by the transformation (33d). After determining the boundary value, evaluate the $\hat{W}$ hypersurface equation (62) using the previously calculated hypersurface values in the partially flat coordinates $\hat{J}, \hat{\beta}$, $\hat{Q}$, and $\hat{U}$.

(vii) Determine the partially flat boundary data $\left.\hat{H}\right|_{\Gamma}$ using (B3), and then evaluate the $\hat{H}$ hypersurface equation (65) using the previously calculated $\hat{J}, \hat{\beta}$, $\hat{Q}, \hat{U}$, and $\hat{W}$.

(viii) Evolve $\hat{J}$ using $\hat{H} \equiv \partial_{\hat{u}} \hat{J}$.

(ix) Evolve $\hat{x}^{\hat{A}}(u)$ using $\partial_{u} \hat{x}^{\hat{A}}=-U^{(0) B} \partial_{B} \hat{x}^{\hat{A}}$.

(x) Iterate to subsequent time steps starting at item 1 of the evolution procedure.

\section{NEWMAN-PENROSE WEYL SCALARS FROM BONDI OR BONDI-LIKE METRIC}

Having determined the metric throughout the compactified null region with the CCE algorithm described in Sec. V above, we are now in a position to compute gauge-invariant (up to BMS) quantities that describe the dynamics of the spacetime at $\mathcal{I}^{+}$. In this section, we give an explicit dictionary between the complementary Bondi-Sachs and Newman-Penrose formalisms. Then, we take advantage of the relation between the asymptotic Weyl scalars in the Newman-Penrose formalism and the spin-weighted scalars from the Bondi-like metric to obtain simple expressions for the asymptotically leading contributions to the Weyl scalars in our partially flat gauge. The result is a simple prescription for adding the full set of Weyl scalars to the outputs of the CCE algorithm.

\section{A. Newman-Penrose tetrad for Bondi-like coordinates}

To calculate the spin coefficients and Weyl scalars, we must select a reasonable choice of orthonormal null tetrad. We adopt a tetrad motivated by [40], but modified with phase $\left(m^{\mu} \rightarrow e^{i \delta} m^{\mu}\right)$ and overall factor $\left(n^{\mu} \rightarrow A n^{\mu}\right.$ and $\left.l^{\mu} \rightarrow l^{\mu} / A\right)$ to asymptotically match tetrads frequently used in numerical relativity [44]:

$$
\begin{aligned}
m^{\mu} & =-\frac{1}{\sqrt{2} r}\left(\sqrt{\frac{K+1}{2}} q^{\mu}-\sqrt{\frac{1}{2(1+K)}} J \bar{q}^{\mu}\right), \\
n^{\mu} & =\sqrt{2} e^{-2 \beta}\left(\delta^{\mu}{ }_{u}-\frac{V}{2 r} \delta^{\mu}{ }_{r}+\frac{1}{2} \bar{U} q^{\mu}+\frac{1}{2} U \bar{q}^{\mu}\right), \\
l^{\mu} & =\frac{1}{\sqrt{2}} \delta^{\mu}{ }_{r} .
\end{aligned}
$$

We emphasize that while these tetrads asymptotically match those often used in numerical relativity, this does not mean that all of the Newman-Penrose spin coefficients and Weyl scalars will similarly match. The subleading in powers of $1 / r$ corrections to the tetrads can cause subtle 
alterations to the scalar functions, so care must be taken to ensure consistent conventions when comparing calculations. More details about the variety of Newman-Penrose conventions in numerical calculations can be found in [45]. Here the spin-weighted scalars $\beta, U, V, J$, and $K$ are assumed to be in generic Bondi-like coordinates, but the results of this section apply to more restrictive coordinates such as Bondi-Sachs or partially flat coordinates; in more restrictive coordinates many of the results simplify further. The tetrads (VI A) satisfy the standard normalization and orthogonality conditions

$$
\begin{aligned}
-l^{\mu} n_{\mu} & =m^{\mu} \bar{m}_{\mu}=1, \\
l^{\mu} l_{\mu} & =l^{\mu} m_{\mu}=n^{\mu} n_{\mu}=n^{\mu} m_{\mu}=m^{\mu} m_{\mu}=0 .
\end{aligned}
$$

From (82), we may write the metric as a combination of these vectors,

$$
g_{\mu \nu}=-2 l_{(\mu} n_{\nu)}+2 m_{(\mu} \bar{m}_{\nu)} .
$$

In addition, it is convenient to define a set of scalar covariant derivatives in terms of the Newman-Penrose null tetrads:

$$
D=l^{\mu} \nabla_{\mu}, \quad \Delta=n^{\mu} \nabla_{\mu}, \quad \delta=m^{\mu} \nabla_{\mu} .
$$

\section{B. Concise closed-form Newman-Penrose spin coefficients}

The Newman-Penrose covariant analogues of the independent Christoffel symbol components are known as spin coefficients and are defined in terms of the orthonormal null tetrad:

$$
\kappa=-m^{\mu} l^{\nu} \nabla_{\nu} l_{\nu},
$$

$$
\begin{aligned}
\rho & =-m^{\mu} \bar{m}^{\nu} \nabla_{\nu} l_{\mu}, \\
\sigma & =-m^{\mu} m^{\nu} \nabla_{\nu} l_{\mu}, \\
\tau & =-m^{\mu} n^{\nu} \nabla_{\nu} l_{\mu}, \\
\nu & =\bar{m}^{\mu} n^{\nu} \nabla_{\nu} n_{\mu}, \\
\mu & =\bar{m}^{\mu} m^{\nu} \nabla_{\nu} n_{\mu}, \\
\lambda & =\bar{m}^{\mu} \bar{m}^{\nu} \nabla_{\nu} n_{\mu}, \\
\pi & =\bar{m}^{\mu} l^{\nu} \nabla_{\nu} n_{\mu}, \\
\epsilon & =\frac{1}{2}\left(\bar{m}^{\mu} l^{\nu} \nabla_{\nu} m_{\mu}-n^{\mu} l^{\nu} \nabla_{\nu} l_{\mu}\right), \\
\beta_{\mathrm{NP}} & =\frac{1}{2}\left(\bar{m}^{\mu} m^{\nu} \nabla_{\nu} m_{\mu}-n^{\mu} m^{\nu} \nabla_{\nu} l_{\mu}\right), \\
\gamma & =\frac{1}{2}\left(\bar{m}^{\mu} n^{\nu} \nabla_{\nu} m_{\mu}-n^{\mu} n^{\nu} \nabla_{\nu} l_{\mu}\right), \\
\alpha & =\frac{1}{2}\left(\bar{m}^{\mu} \bar{m}^{\nu} \nabla_{\nu} m_{\mu}-n^{\mu} \bar{m}^{\nu} \nabla_{\nu} l_{\mu}\right) .
\end{aligned}
$$

To resolve the notation collision between the Bondi-like $\beta$ that appears in the metric (1) and the Newman-Penrose spin coefficient that is traditionally notated with the same symbol, we write the spin coefficient with a subscript: $\beta_{\mathrm{NP}}$.

It is also convenient to define the unit spherical connection coefficient $\Theta=q^{A} \bar{q}_{B} \nabla_{A} q^{B}$. Under these definitions, the resulting spin-weighted Bondi-like expressions for the Newman-Penrose spin coefficients are

$$
\begin{aligned}
\kappa & =0 \\
\rho & =-\frac{1}{\sqrt{2} r}, \\
\sigma & =-\frac{(1+K) \partial_{r} J}{4 \sqrt{2} K}+\frac{J^{2} \partial_{r} \bar{J}}{4 \sqrt{2} K(1+K)}, \\
\tau & =-\frac{(2 \bar{\jmath} \beta-\bar{Q}) J}{4 r \sqrt{1+K}}+\frac{(2 ð(\beta)-Q) \sqrt{1+K}}{4 r}, \\
\nu & =\frac{ð W \bar{J}}{2 e^{2 \beta} \sqrt{1+K}}-\frac{\bar{\partial} W \sqrt{1+K}}{2 e^{2 \beta}}, \\
\mu & =\frac{e^{-2 \beta}(ð \bar{U}+\bar{\jmath} U)}{2 \sqrt{2}}-\frac{e^{-2 \beta}\left(r^{2} W+r\right)}{\sqrt{2} r^{2}},
\end{aligned}
$$




$$
\begin{aligned}
& \lambda=\frac{e^{-2 \beta}}{\sqrt{2}}\left[\frac{1}{2} \bar{J}(\bar{\jmath} U-ð \bar{U})-\frac{\bar{J}^{2} \text { } U}{2(1+K)}+\frac{\bar{\jmath} \bar{U}(1+K)}{2}+\frac{r^{2} W+r}{r}\left(\frac{\bar{J}^{2} \partial_{r} J}{1+K}-\partial_{r} \bar{J}(1+K)\right)\right. \\
& \left.+\frac{U}{4 K}\left(\bar{\partial} \bar{J}(1+K)-\frac{\bar{J}^{2} \bar{\partial} J}{1+K}\right)+\frac{\bar{U}}{4 K}\left(ð \bar{J}(1+K)-\frac{\bar{J}^{2} \text { } J}{1+K}\right)-\frac{\bar{J}^{2} \partial_{u} J}{2 K(1+K)}+\frac{(1+K) \partial_{u} \bar{J}}{2 K}\right], \\
& \pi=\frac{(2 ð \beta+Q) \bar{J}}{4 r \sqrt{1+K}}-\frac{(2 \bar{ð}(\beta)+\bar{Q}) \sqrt{1+K}}{4 r}, \\
& \epsilon=\frac{\partial_{r}(\beta)}{\sqrt{2}}+\frac{J \partial_{r}(\bar{J})-\bar{J} \partial_{r}(J)}{8 \sqrt{2}(1+K)} \\
& \beta_{\mathrm{NP}}=\frac{1}{4 r \sqrt{1+K}}\left[\bar{\jmath} \beta J-ð \beta(1+K)-\frac{ð(J \bar{J})}{4 K}-\frac{J(\bar{\jmath}(J \bar{J})-\bar{J} \bar{\jmath} J)}{4 K(1+K)}+\frac{\bar{\partial} J(1+3 K)}{4 K}\right. \\
& \left.+\frac{1}{2} J \bar{Q}-\frac{1}{2} Q(1+K)-\frac{1}{2} J \bar{\Theta}-\frac{1}{2}(1+K) \Theta\right] \text {, } \\
& \alpha=\frac{1}{4 r \sqrt{1+K}}\left[\text { б } \beta \bar{J}-\bar{ð} \beta(1+K)+\frac{\bar{\jmath}(J \bar{J})}{4 K}+\frac{\bar{J}(ð(J \bar{J})-J ð \bar{J})}{4 K(1+K)}-\frac{\text { } J(1+3 K)}{4 K}\right. \\
& \left.+\frac{1}{2} \bar{J} Q-\frac{1}{2} \bar{Q}(1+K)+\frac{1}{2} \bar{J} \Theta+\frac{1}{2}(1+K) \bar{\Theta}\right] \\
& \gamma=\frac{e^{-2 \beta}}{\sqrt{2}}\left[\frac{1}{4}(J \bar{ð} \bar{U}-\bar{J} ð U)+\frac{K}{4}(\bar{\partial} U-ð \bar{U})+\frac{r^{2} W+r}{8 r(1+K)}\left(\bar{J} \partial_{r} J-J \partial_{r} \bar{J}\right)+\frac{1}{2}\left(r \partial_{r} W+W\right)\right. \\
& \left.+\frac{U(\bar{\partial}(J \bar{J})-2 \bar{J} \bar{\partial} J)}{8(1+K)}-\frac{\bar{U}(ð(J \bar{J})-2 J ð \bar{J})}{8(1+K)}-\frac{\bar{J} \partial_{u} J}{4(1+K)}+\frac{J \partial_{u} \bar{J}}{4(1+K)}+\frac{1}{4} \bar{U} \Theta-\frac{1}{4} U \bar{\Theta}\right] .
\end{aligned}
$$

Note that the Newman-Penrose spin coefficients themselves involve no time derivatives beyond those that would be computed during the Bondi coordinate characteristic evolution, so they may be computed in a practical numerical implementation with little trouble.

\section{Computation of Weyl scalars}

Having established the spin coefficients in terms of Bondi quantities, we can easily derive the Weyl scalars in the Newman-Penrose formalism by taking advantage of a selected subset of the Newman-Penrose equations,

$$
\begin{aligned}
\Psi_{0}= & D \sigma-\delta \kappa-(\rho+\bar{\rho}) \sigma-(3 \epsilon-\bar{\epsilon}) \sigma \\
& +\left(\tau-\bar{\pi}+\bar{\alpha}+3 \beta_{\mathrm{NP}}\right) \kappa, \\
\Psi_{1}= & D \beta_{\mathrm{NP}}-\delta \epsilon-(\alpha+\pi) \sigma-(\bar{\rho}-\bar{\epsilon}) \beta_{\mathrm{NP}} \\
& +(\mu+\gamma) \kappa+(\bar{\alpha}-\bar{\pi}) \epsilon, \\
\Psi_{2}= & D \mu-\delta \pi-(\bar{\rho}-\epsilon-\bar{\epsilon}) \mu-\sigma \lambda \\
& +\left(\bar{\alpha}-\beta_{\mathrm{NP}}-\bar{\pi}\right) \pi+\nu \kappa, \\
\Psi_{3}= & D \nu-\Delta \pi-(\pi+\bar{\tau}) \mu-(\bar{\pi}+\tau) \lambda \\
& -(\gamma-\bar{\gamma}) \pi+(3 \epsilon+\bar{\epsilon}) \nu,
\end{aligned}
$$

$$
\begin{aligned}
\Psi_{4}= & -\Delta \lambda+\bar{\delta} \nu-\lambda(\mu+\bar{\mu})-(3 \gamma-\bar{\gamma}) \lambda \\
& +\left(3 \alpha+\bar{\beta}_{\mathrm{NP}}+\pi-\bar{\tau}\right) \nu
\end{aligned}
$$

where we have specialized to a vacuum solution, setting $R_{\mu \nu}=0$.

While some of the spin coefficients depend on the dyad connection $\Theta$, the coordinate invariance of the Weyl scalars ensures that all such contributions must cancel or produce dependence on the gauge-invariant spherical curvature scalar in the calculation of the Weyl scalars. This identity is used as a check on the spin coefficients' calculation in the accompanying Mathematica document [39].

Most of the Newman-Penrose spin coefficients in (VI B) are spin-weighted scalars in the Bondi-like system. The exceptions are $\beta_{\mathrm{NP}}, \alpha$, and $\gamma$, which have explicit coordinate dependence via the connection terms $\Theta$ and $\bar{\Theta}$. We therefore introduce the more convenient method of defining new spin-weighted scalars $\beta_{\mathrm{NP}}^{\mathrm{SW}}=\left.\beta\right|_{\Theta=0}, \alpha^{\mathrm{SW}}=\left.\alpha\right|_{\Theta=0}$, and $\gamma^{\mathrm{SW}}=\left.\gamma\right|_{\Theta=0}$. Then, we define the spin-weighted generalizations of the scalar derivatives:

$$
\Delta^{\mathrm{SW}}=\sqrt{2} e^{-2 \beta}\left(\left(\delta^{\mu}{ }_{u}-\frac{V}{2 r} \delta_{r}^{\mu}\right) \nabla_{\mu}+\frac{1}{2} \bar{U} ð+\frac{1}{2} U \bar{ð}\right),
$$




$$
\delta^{\mathrm{SW}}=-\frac{1}{\sqrt{2} r}\left(\sqrt{\frac{K+1}{2}} \text { ð }-\frac{1}{2(1+K)} J \bar{ð}\right) .
$$

The Weyl scalar identities (VIC) remain unchanged under the replacements

$$
\left\{\beta_{\mathrm{NP}}, \alpha, \gamma, \Delta, \delta\right\} \rightarrow\left\{\beta_{\mathrm{NP}}^{\mathrm{SW}}, \alpha^{\mathrm{SW}}, \gamma^{\mathrm{SW}}, \Delta^{\mathrm{SW}}, \delta^{\mathrm{SW}}\right\}
$$

Therefore, the most direct route to calculating $\Psi_{2}, \Psi_{3}$, and $\Psi_{4}$ in the bulk of the spacetime is to perform the above replacements of the spin coefficients and derivatives, obtaining

$$
\begin{aligned}
\Psi_{2}= & \partial_{r} \mu+\frac{1}{2 r}\left(\frac{J \bar{\partial} \pi}{\sqrt{1+K}}-\sqrt{1+K} \text { ð } \pi\right)+(\bar{\epsilon}+\epsilon-\bar{\rho}) \mu+\left(\bar{\alpha}^{\mathrm{SW}}-\bar{\pi}-\beta_{\mathrm{NP}}^{\mathrm{SW}}\right) \pi-\lambda \sigma, \\
\Psi_{3}= & \frac{1}{\sqrt{2}} \partial_{r} \nu-\sqrt{2} e^{-2 \beta} \partial_{u} \pi+\frac{e^{-2 \beta}}{\sqrt{2}}(r W+1)\left(\partial_{r} \pi-U \bar{\partial} \pi-\bar{U} \partial \pi\right) \\
& -(\pi+\bar{\tau}) \mu-(\bar{\pi}+\tau) \lambda-\left(\gamma^{\mathrm{SW}}-\bar{\gamma}^{\mathrm{SW}}\right) \pi+(3 \epsilon+\bar{\epsilon}) \nu, \\
\Psi_{4}= & \frac{1}{2 r}\left(\sqrt{1+K} \bar{\partial} \nu-\frac{\bar{J} \partial \nu}{\sqrt{1+k}}\right)-e^{-2 \beta} \partial_{u} \lambda+\frac{e^{-2 \beta}}{2}(r W+1)\left(\partial_{r} \lambda-U \bar{\partial} \lambda-\bar{U} \partial \lambda\right) \\
& -\left(\mu+\bar{\mu}+3 \gamma^{\mathrm{SW}}-\bar{\gamma}^{\mathrm{SW}}\right) \lambda+\left(3 \alpha^{\mathrm{SW}}+\bar{\beta}_{\mathrm{NP}}^{\mathrm{SW}}+\pi-\bar{\tau}\right) \nu .
\end{aligned}
$$

These can easily be expanded using the previous definitions, and the full expression in terms of spin-weighted quantities is given in the accompanying Mathematica document [39]. However, the results do not give interesting simplifications. The quantities $\Psi_{0}$ and $\Psi_{1}$, however, possess cancellations that allow for a concise full expression in terms of Bondi quantities

$$
\begin{aligned}
\Psi_{0}= & \left(\frac{r \partial_{r} \beta-1}{4 K r}\right)\left((1+K) \partial_{r} J-\frac{J^{2} \partial_{r} \bar{J}}{(1+K)}\right)+\frac{J\left(1+K^{2}\right) \partial_{r} J \partial_{r} \bar{J}}{8 K^{3}} \\
& +\frac{1}{8 K}\left(\frac{J^{2} \partial_{r}^{2} \bar{J}}{1+K}-(1+K) \partial_{r}^{2} J\right)+\frac{-J \bar{J}^{2} \partial_{r} J^{2}+J^{3} \partial_{r} \bar{J}^{2}}{16 K^{3}}, \\
\Psi_{1}= & \frac{1}{4 \sqrt{2(K+1) r}}\left(-J \partial_{r}(2 \bar{\jmath} \beta-\bar{Q})+\left(\frac{J^{2} \partial_{r} \bar{J}-(1+K) \partial_{r} J}{4 K}+\frac{J}{r}\right)(2 \bar{\jmath} \beta+\bar{Q})\right. \\
& \left.-(1+K) \partial_{r}(2 ð \beta-Q)-\left(\frac{1+K}{r}+\frac{(1+K) \bar{J} \partial_{r} J}{4 K}-\frac{J^{2} \bar{J} \partial_{r} \bar{J}}{4 K(1+K)}\right)(2 ð \beta+Q)\right) .
\end{aligned}
$$

\section{Asymptotic Weyl scalars in partially flat gauge}

The full set of Weyl scalars evaluated at $\mathcal{I}^{+}$would provide detailed, gauge-invariant (up to tetrad ambiguity) information about the dynamical spacetime. It is therefore valuable to describe the computation of the leading asymptotic contribution for each Weyl scalar in the partially flat gauge presented in this paper, as well as the equivalent expressions in a true BondiSachs gauge. According to the peeling theorem, the radial falloff of the Weyl scalars obeys

$$
\Psi_{n} \sim r^{n-5}
$$

In a general gauge, the form of the Weyl scalars cannot be significantly simplified beyond an asymptotic expansion of (90) and (91) in powers of $r^{-1}$. However, in the partially flat gauge (see Sec. III), substantial simplifications are available. Applying the partially flat gauge conditions and components of the Einstein field equations, we find

$$
\begin{aligned}
& \lim _{\hat{r} \rightarrow \infty} \hat{r}^{5} \Psi_{0}^{\mathrm{PF}}=\frac{3}{2}\left(\frac{1}{4} \hat{\bar{J}}^{(1)} \hat{J}^{(1) 2}-\hat{J}^{(3)}\right), \\
& \lim _{\hat{r} \rightarrow \infty} \hat{r}^{4} \Psi_{1}^{\mathrm{PF}}=\frac{1}{8}\left(-12 \hat{\jmath} \hat{\beta}^{(2)}+\hat{J}^{(1)} \hat{\bar{Q}}^{(1)}+2 \hat{Q}^{(2)}\right),
\end{aligned}
$$




$$
\begin{aligned}
& \lim _{\hat{r} \rightarrow \infty} \hat{r}^{3} \Psi_{2}^{\mathrm{PF}}=-\frac{e^{-2 \hat{\beta}^{(0)}}}{4}\left(e^{2 \hat{\beta}^{(0)}} \text { ð} \hat{\bar{Q}}^{(1)}+\hat{\partial} \hat{\bar{U}}^{(2)}+\hat{\bar{\partial}} \hat{U}^{(2)}+\hat{J}^{(1)} \hat{\bar{\partial}} \hat{\bar{U}}^{(1)}+\hat{J}^{(1)} \hat{\bar{H}}^{(1)}-2 \hat{W}^{(2)}\right), \\
& \lim _{\hat{r} \rightarrow \infty} \hat{r}^{2} \Psi_{3}^{\mathrm{PF}}=2 \hat{\bar{\partial}} \hat{\beta}^{(0)}+4 \hat{\bar{\partial}} \hat{\beta}^{(0)} \hat{\bar{\partial}} \hat{\partial} \hat{\beta}^{(0)}+\hat{\bar{\partial}} \hat{\bar{\partial}} \hat{\partial} \hat{\beta}^{(0)}+\frac{e^{-2 \hat{\beta}^{(0)}}}{2} \hat{\partial} \hat{\bar{H}}^{(1)}-e^{-2 \hat{\beta}^{(0)}} \hat{\partial} \hat{\beta}^{(0)} \hat{\bar{H}}^{(1)}, \\
& \lim _{\hat{r} \rightarrow \infty} \hat{r} \Psi_{4}^{\mathrm{PF}}=-e^{-2 \hat{\beta}^{(0)}} \partial_{\hat{u}}\left[e^{-2 \hat{\beta}^{(0)}}\left(\hat{\bar{\partial}} \hat{\bar{U}}^{(1)}+\hat{\bar{H}}^{(1)}\right)\right] .
\end{aligned}
$$

In a full Bondi-Sachs gauge, the leading Weyl scalars can be simplified somewhat further, giving

$$
\begin{aligned}
& \lim _{r \rightarrow \infty} \stackrel{\circ}{r}^{5} \Psi_{0}^{\text {Bondi }}=\frac{3}{2}\left(\frac{1}{4} \stackrel{\circ}{J}^{(1) \circ(1) 2}-\stackrel{\circ}{J}(3)\right) \\
& =\Psi_{0}^{\mathrm{PF}(5)}(\hat{u}(\stackrel{\circ}{u}))+2 \hat{\partial} \stackrel{\circ}{u} \Psi_{1}^{\mathrm{PF}(4)}(\hat{u}(\stackrel{\circ}{u}))+\frac{3}{4}\left(\hat{\jmath}^{\circ}\right)^{2} \Psi_{2}^{\mathrm{PF}(3)}(\hat{u}(\stackrel{\circ}{u})) \\
& +\frac{1}{2}(\hat{\partial} \stackrel{\circ}{u})^{3} \Psi_{3}^{\mathrm{PF}(2)}(\hat{u}(\stackrel{\circ}{u}))+\frac{1}{16}\left(\hat{\jmath}^{\circ}\right)^{4} \Psi_{4}^{\mathrm{PF}(1)}(\hat{u}(\stackrel{\circ}{u})), \\
& \lim _{r \rightarrow \infty} \stackrel{\circ}{r}^{4} \Psi_{1}^{\text {Bondi }}=\frac{1}{8}\left(-12 \stackrel{\circ}{\partial} \beta^{(2)}+\stackrel{\circ}{J}^{(1)} \stackrel{\circ}{Q}^{(1)}+2 \stackrel{\circ}{Q}^{(2)}\right) \\
& =\Psi_{1}^{\mathrm{PF}(4)}(\hat{u}(\stackrel{\circ}{u}))+\frac{3}{2} \hat{\jmath} \stackrel{\circ}{u} \Psi_{2}^{\mathrm{PF}(3)}(\hat{u}(\stackrel{\circ}{u}))+\frac{3}{4}\left(\hat{\jmath}^{\circ}\right)^{2} \Psi_{3}^{\mathrm{PF}(3)}(\hat{u}(\stackrel{\circ}{u}))+\frac{1}{8}\left(\hat{\jmath}^{\circ}\right)^{3} \Psi_{4}^{\mathrm{PF}(1)}(\hat{u}(\stackrel{\circ}{u})), \\
& \lim _{r \rightarrow \infty} \stackrel{\circ}{r}^{3} \Psi_{2}^{\text {Bondi }}=\frac{\stackrel{\circ}{W}^{(2)}}{2}-\frac{1}{8} \stackrel{\circ}{\partial} \stackrel{\circ}{\partial} \bar{J}^{(1)}+\frac{1}{8} \stackrel{\circ}{\bar{\partial}} \check{\circ}^{\partial} \stackrel{\circ}{J}^{(1)}-\frac{1}{4} \stackrel{\circ}{ }^{(1)} \stackrel{\circ}{H}^{(1)} \\
& =\Psi_{2}^{\mathrm{PF}(3)}(\hat{u}(\stackrel{\circ}{u}))+\hat{\jmath}^{\circ} \Psi_{3}^{\mathrm{PF}(2)}(\hat{u}(\stackrel{\circ}{u}))+\frac{1}{4}\left(\hat{\jmath}^{\circ} \stackrel{\circ}{u}\right)^{2} \Psi_{4}^{\mathrm{PF}(1)}(\hat{u}(\stackrel{\circ}{u})), \\
& \lim _{r \rightarrow \infty} \stackrel{\circ}{r}^{2} \Psi_{3}^{\text {Bondi }}=\frac{1}{2} \stackrel{\circ}{\partial} \bar{H}^{(1)} \\
& =\Psi_{3}^{\mathrm{PF}(2)}(\hat{u}(\stackrel{\circ}{u}))+\frac{1}{2} \hat{\partial}^{\circ}{ }^{(0)} \Psi_{4}^{\mathrm{PF}(1)}(\hat{u}(\stackrel{\circ}{u})), \\
& \lim _{r \rightarrow \infty} \stackrel{\circ}{r} \Psi_{4}^{\text {Bondi }}=-\partial_{u} \stackrel{\circ}{H}^{(1)} \\
& =\Psi_{4}^{\mathrm{PF}(1)}(\hat{u}(\stackrel{\circ}{u})) .
\end{aligned}
$$

In the second line of each of the above identities, we have included the relationship to the Weyl scalars computed in the partially flat coordinates. These relations are the transformations given in [46], adapted to our conventions for $ð$ and Newman-Penrose tetrads.

\section{CONCLUSIONS}

In this paper, we have laid the formulation groundwork for the next generation of Cauchy-characteristic evolution code. Spectral methods have shown that they give an efficient treatment of a fully nonlinear asymptotic characteristic system [31] in general relativity. However, they have previously suffered from logarithmic dependence that threatens the numerical precision of polynomial-based spectral techniques. We have streamlined the previously derived [21,28] characteristic evolution equations for ease and efficiency of numerical implementation (Sec. IV). We have demonstrated that there exists a computationally simple "partially flat" gauge (Sec. III) that provably avoids any logarithmic dependence (Sec. V). We have also provided a direct roadmap (Sec. V C) for the numerical implementation of our suggested method. 
In the process of determining the computationally preferred partially flat gauge, we have also extended the coordinate methods for interfacing between a generic coordinate system and the highly fixed Bondi-Sachs coordinates. When taken together with previous results [24] that determine a Bondi-like metric (4) from an arbitrary coordinate system, our coordinate transformations in Sec. III determine the corresponding Bondi-Sachs metric, which is unique up to residual BMS freedom.

Finally, we have demonstrated the significant simplifications given by using the partially flat gauge for the determination of asymptotic quantities. We provide simple formulas for computing the leading contributions to each of the Weyl scalars (Sec. VI) and the Bondi news (Sec. III B).

The most direct extension of this work is the numerical implementation itself, which is underway as a module of the SpECTRE [34] simulation codebase. In forthcoming work, we will present the performance and precision tests of the implementation.

The ability to determine asymptotic quantities to high numerical precision without gauge ambiguities will have numerous applications. It can be used to develop improved waveform models for LIGO data analysis. It can also be used for investigating various theoretical issues in general relativity involving quantities at $\mathcal{I}^{+}$, including memory effects.

In future work, we will also explore the use of the methods presented in this paper in a Cauchy-characteristic matching (CCM) scheme. CCM uses the nonlinear characteristic evolution to provide a fully physical vacuum boundary condition for the Cauchy code. CCM, however, involves the considerable complexity of running an efficient characteristic evolution in tandem, and in communication with, a Cauchy code $[24,47]$. Because of the technical challenges, there have been no implementations of nonlinear CCM, but the formulation advances presented in this work will ease the way to a successful matching system.

\section{ACKNOWLEDGMENTS}

We thank Kevin Barkett, Michael Boyle, Dante Iozzo, Bela Szilagyi, and Jeff Winicour for valuable discussions and suggestions regarding this project. We thank Harald Pfeiffer for suggestions improving the presentation in this paper. This work was supported in part by the Sherman Fairchild Foundation and by NSF Grants No. PHY1708212, No. PHY-1708213, and No. OAC-1931266 at Caltech and NSF Grants No. PHY-1912081 and No. OAC1931280 at Cornell.

\section{APPENDIX A: COORDINATE SYSTEM GLOSSARY}

Because of the ease of generating subtle mistakes or misunderstandings when using multiple similar coordinate systems, we are careful throughout this paper to introduce a complete coordinate system with distinct index style each time a coordinate transformation is suggested. In scenarios involving partial derivatives, the coordinate notation carries the implication of holding fixed all remaining coordinates of a given set. The need to clearly express derivatives motivates our extensive notation. For instance, the Cauchy coordinates and Bondi-like coordinates share time and angular coordinates $u=t^{\prime}, x^{A}=\delta_{A^{\prime}}^{A} x^{\prime A^{\prime}}$, but because of the differing radial coordinates, the partial derivatives with respect to time and angles are not the same, e.g., $g_{A B, u} \neq g_{A^{\prime} B^{\prime}, t^{\prime}}$. The full collection of our coordinate system notation is given in Table I.

TABLE I. Coordinate and index notation used in our presentation of the CCE formalism.

\begin{tabular}{|c|c|c|c|c|}
\hline Coordinate system & Coordinates & $\begin{array}{l}\text { Index } \\
\text { style }\end{array}$ & $\begin{array}{l}\text { Sections } \\
\text { used }\end{array}$ & Explanation \\
\hline Cauchy coordinates & $\left\{t^{\prime}, r^{\prime}, x^{\prime A^{\prime}}\right\}$ & $\alpha^{\prime}$ & II & Input coordinates on the world tube, provided by a Cauchy simulation. \\
\hline Radial null coordinates & $\left\{\underline{u}, \underline{\lambda}, \underline{x}^{A}\right\}$ & $\underline{\alpha}$ & II B & $\begin{array}{l}\text { Intermediate step in deriving Bondi-like coordinates. } \\
\text { Satisfies } g_{\underline{\lambda} \underline{\lambda}}=g_{\underline{\lambda}} \underline{A}=0\end{array}$ \\
\hline Bondi-like coordinates & $\left\{u, r, x^{A}\right\}$ & $\alpha$ & II-VI & $\begin{array}{l}\text { Coordinates in evolution of standard CCE algorithm. } \\
\text { Satisfies } g_{r r}=g_{r A}=0 \text { and } \operatorname{det}\left(g_{A B}\right)=\operatorname{det}\left(q_{A B}\right) \text {. }\end{array}$ \\
\hline $\begin{array}{l}\text { Partially flat Bondi-like } \\
\text { coordinates }\end{array}$ & $\left\{\hat{u}, \hat{r}, \hat{x}^{\hat{A}}\right\}$ & $\hat{\alpha}$ & III, V, VI & $\begin{array}{l}\text { Partially restricted Bondi-like coordinates, preferred for use } \\
\text { in computational schemes described in this paper. } \\
\text { Satisfies } g_{\hat{r} \hat{r}}=g_{\hat{r} \hat{A}}=0, \operatorname{det}\left(g_{\hat{A} \hat{B}}\right)=\operatorname{det}\left(q_{\hat{A} \hat{B}}\right) \text {, and all metric } \\
\text { components except for } \hat{B} \text { asymptotically approach Minkowski form. }\end{array}$ \\
\hline Bondi coordinates & $\left\{\stackrel{\circ}{u}, \stackrel{\circ}{r}, \stackrel{\circ}{x}{ }^{\AA}\right\}$ & $\stackrel{\circ}{\alpha}$ & II, III, VI & $\begin{array}{l}\text { Bondi-Sachs coordinates, satisfying } g_{r r}^{\circ}=g_{r A}=0, \operatorname{det} g_{A B}^{\circ}=\operatorname{det} q_{A B} \text {, } \\
\text { and asymptotically approaches Minkowski as per (3). }\end{array}$ \\
\hline $\begin{array}{l}\text { Numerically adapted } \\
\text { coordinates }\end{array}$ & $\{\breve{u}, \breve{y}, \breve{x} \breve{A}\}$ & $\breve{\alpha}$ & IV & $\begin{array}{l}\text { Coordinates associated with a generic Bondi or Bondi-like coordinate } \\
\text { system (which can include the partially flat or true Bondi-Sachs } \\
\text { coordinates), but with a numerically adapted radial coordinate } \\
\breve{y} \in[-1,1] \text {. }\end{array}$ \\
\hline
\end{tabular}




\section{APPENDIX B: ADDITIONAL TRANSFORMATIONS TO PARTIALLY FLAT COORDINATES}

Here we give detailed transformations for $\hat{Q}$ and $\hat{H}$ in a form amenable to numerical implementation for boundary computations described in Sec. V.

$\hat{Q}$ may be obtained from $\partial_{\hat{r}} \hat{U}$ as

$$
\hat{Q}=\hat{r}^{2} e^{-2 \hat{\beta}}\left(\hat{K} \partial_{\hat{r}} \hat{U}+\hat{J} \partial_{\hat{r}} \hat{\bar{U}}\right)
$$

and

$$
\begin{aligned}
\partial_{\hat{r}} \hat{U}= & \frac{1}{2 \hat{\omega}^{3}}\left(\hat{\bar{b}} \partial_{r} U-\hat{a} \partial_{r} \bar{U}\right)+\frac{e^{2 \hat{\beta}}}{4 \hat{\omega}}(\hat{J} \hat{\bar{\partial}} \hat{\omega}-\hat{K} \hat{\jmath} \hat{\omega})\left(\partial_{\hat{r}} \hat{\bar{J}} \partial_{\hat{r}} \hat{J}-\frac{\partial_{\hat{r}}(\hat{J} \hat{\bar{J}})^{2}}{4 \hat{K}^{2}}\right) \\
& +\frac{e^{2 \hat{\beta}}}{\hat{\omega} \hat{r}}\left[\hat{\bar{\partial}} \hat{\omega}\left(\partial_{\hat{r}} \hat{J}-\frac{\hat{J}}{\hat{r}}\right)+\hat{\bar{\partial}} \hat{\omega}\left(\frac{\hat{K}}{\hat{r}}-\frac{\hat{J} \partial_{\hat{r}} \hat{\bar{J}}+\hat{\bar{J}} \partial_{\hat{r}} \hat{J}}{2 \hat{K}}\right)\right] .
\end{aligned}
$$

In terms of other transformed quantities, $\hat{H}$ is

$$
\begin{aligned}
\hat{H}= & \frac{\partial_{\hat{u}} \hat{\omega}-\frac{1}{2}\left(\mathcal{U}^{(0)} \overline{\widehat{\partial}} \hat{\omega}+\overline{\mathcal{U}}^{(0)} \hat{\jmath} \hat{\omega}\right)}{\hat{\omega}}\left(2 \hat{J}-\hat{r} \partial_{\hat{r}} \hat{J}\right)-\hat{J} \hat{\bar{\partial}} \mathcal{U}^{(0)}+\hat{K} \hat{\partial} \overline{\mathcal{U}}^{(0)} \\
& +\frac{1}{4 \hat{\omega}}\left(\hat{\bar{b}}^{2} H+\hat{a}^{2} \bar{H}+\hat{\bar{b}} a \frac{H \bar{J}+J \bar{H}}{K}\right)+\frac{1}{2}\left(\mathcal{U}^{(0)} \hat{\bar{\partial}} \hat{J}+\hat{\jmath}\left(\overline{\mathcal{U}}^{(0)} \hat{J}\right)-\hat{J} \hat{\partial} \overline{\mathcal{U}}^{(0)}\right) .
\end{aligned}
$$

\section{APPENDIX C: PERTURBATIVE EXPANSION OF THE TRANSFORMATIONS TO BONDI-SACHS COORDINATES NEAR $\mathcal{I}^{+}$}

Here we give the first few orders of the asymptotic coordinate transformations necessary to move from partially flat coordinates to Bondi-Sachs coordinates. These expressions are expanded from the equations derived in Sec. III.

Perturbative expansion of the defining equation for $\stackrel{\circ}{u}=\stackrel{\circ}{u^{(0)}}+\hat{l}^{\circ}(1)+\hat{l}^{2} \stackrel{\circ}{u^{(2)}}+\mathcal{O}\left(\hat{l}^{3}\right)$ in (36) gives rise to the equations

$$
\begin{gathered}
2 \dot{u}^{(1)}=-ð{ }^{(0)} \bar{\partial} u^{(0)}, \\
-4 u^{(2)}-2 e^{-2 \hat{\beta}} \partial_{\hat{u}} u^{(1)} u^{(1)}=2 \partial_{\hat{A}}^{\circ} u^{(0)} \stackrel{\circ}{u}^{(1)} \hat{U}^{(1) \hat{A}} e^{-2 \hat{\beta}}-\left(\bar{ð}^{\circ} u^{(0)}\right)^{2} J^{(1)}-\left(ð u^{\circ}(0)\right)^{2} \bar{J}^{(1)} .
\end{gathered}
$$

Perturbative expansion of $\stackrel{\circ}{x}^{\circ}=\delta^{\AA}{ }_{\hat{A}} \hat{x}^{\hat{A}}+\hat{l}^{\circ} x^{(1) \stackrel{\circ}{ }}+\hat{l}^{\circ} x^{\circ(2) \stackrel{\circ}{A}}+\mathcal{O}\left(\hat{l}^{3}\right)$ in (38) gives the equations

$$
\begin{aligned}
& -\stackrel{\circ}{x}(1) \stackrel{\circ}{A}=\hat{q}^{\hat{A}} \hat{B} \partial_{\hat{\beta}} u^{(0)},
\end{aligned}
$$

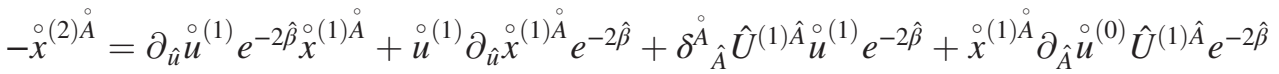

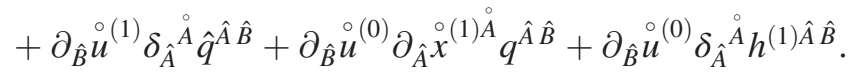

Finally, the leading alteration to the conformal factor $\stackrel{\circ}{\omega}=1+\hat{l}_{\omega}^{\circ}(1)+\mathcal{O}\left(\hat{l}^{2}\right)$ is

$$
\stackrel{\circ}{\omega}^{(1)}=\frac{1}{2} \hat{\partial} \hat{\jmath}^{\circ}(0) .
$$


[1] B. P. Abbott et al. (LIGO Scientific and Virgo Collaborations), Phys. Rev. Lett. 116, 061102 (2016).

[2] B. P. Abbott et al. (LIGO Scientific and Virgo Collaborations), Phys. Rev. Lett. 116, 241103 (2016).

[3] B. P. Abbott et al. (LIGO Scientific and Virgo Collaborations), Phys. Rev. X 9, 031040 (2019).

[4] B. P. Abbott et al. (KAGRA, LIGO Scientific and Virgo Collaborations), Living Rev. Relativity 21, 3 (2018).

[5] C. Hanna and (LIGO Scientific Virgo Collaborations), Classical Quantum Gravity 27, 114003 (2010).

[6] E. E. Flanagan and S. A. Hughes, Phys. Rev. D 57, 4535 (1998).

[7] B. P. Abbott et al. (LIGO Scientific and Virgo Collaborations), Phys. Rev. D 94, 064035 (2016).

[8] P. Kumar, J. Blackman, S. E. Field, M. Scheel, C. R. Galley, M. Boyle, L. E. Kidder, H. P. Pfeiffer, B. Szilagyi, and S. A. Teukolsky, Phys. Rev. D 99, 124005 (2019).

[9] P. Kumar, K. Barkett, S. Bhagwat, N. Afshari, D. A. Brown, G. Lovelace, M. A. Scheel, and B. Szilagyi, Phys. Rev. D 92, 102001 (2015).

[10] J. e. Lange, Phys. Rev. D 96, 104041 (2017).

[11] G. Lovelace et al., Classical Quantum Gravity 33, 244002 (2016).

[12] B. P. Abbott et al. (LIGO Scientific and Virgo Collaborations), Phys. Rev. D 100, 104036 (2019).

[13] M. Isi, M. Giesler, W. M. Farr, M. A. Scheel, and S. A. Teukolsky, Phys. Rev. Lett. 123, 111102 (2019).

[14] The Spectral Einstein Code, https://www.black-holes.org/ code/spec.html.

[15] F. Loffler et al., Classical Quantum Gravity 29, 115001 (2012).

[16] B. Brugmann, W. Tichy, and N. Jansen, Phys. Rev. Lett. 92, 211101 (2004).

[17] I. Ruchlin and Z. B. Etienne, and T. W. Baumgarte, Phys. Rev. D 97, 064036 (2018).

[18] M. Boyle and A. H. Mroue, Phys. Rev. D 80, 124045 (2009).

[19] T. Chu, H. Fong, P. Kumar, H. P. Pfeiffer, M. Boyle, D. A. Hemberger, L. E. Kidder, M. A. Scheel, and B. Szilagyi, Classical Quantum Gravity 33, 165001 (2016).

[20] N. T. Bishop, R. Gomez, L. Lehner, and J. Winicour, Phys. Rev. D 54, 6153 (1996).

[21] N. T. Bishop, R. Gomez, L. Lehner, M. Maharaj, and J. Winicour, Phys. Rev. D 56, 6298 (1997).

[22] J. Winicour, Living Rev. Relativity 15, 2 (2012).

[23] M. C. Babiuc, J. Winicour, and Y. Zlochower, Classical Quantum Gravity 28, 134006 (2011).
[24] N. T. Bishop, R. Gomez, L. Lehner, B. Szilagyi, J. Winicour, and R. A. Isaacson, in Black Holes, Gravitational Radiation and the Universe: Essays in Honor of C.V. Vishveshwara, edited by B. R. Iyer and B. Bhawal (Springer Netherlands, 1998), pp. 383-408.

[25] C. Reisswig, N. Bishop, D. Pollney, and B. Szilagyi, Classical Quantum Gravity 27, 075014 (2010).

[26] C. Reisswig, N. T. Bishop, D. Pollney, and B. Szilagyi, Phys. Rev. Lett. 103, 221101 (2009).

[27] M. C. Babiuc, B. Szilagyi, J. Winicour, and Y. Zlochower, Phys. Rev. D 84, 044057 (2011).

[28] C. J. Handmer and B. Szilagyi, Classical Quantum Gravity 32, 025008 (2015).

[29] C. J. Handmer, B. Szilágyi, and J. Winicour, Classical Quantum Gravity 32, 235018 (2015).

[30] C. J. Handmer, B. Szilágyi, and J. Winicour, Classical Quantum Gravity 33, 225007 (2016).

[31] K. Barkett, J. Moxon, M. A. Szilágyi, and B. Scheel, Phys. Rev. D 102, 024004 (2020).

[32] P. D. Lasky, E. Thrane, Y. Levin, J. Blackman, and Y. Chen, Phys. Rev. Lett. 117, 061102 (2016).

[33] J. Moxon, M. Scheel, and S. Teukolsky (to be published)..

[34] L. E. Kidder et al., J. Comput. Phys. 335, 84 (2017).

[35] H. Bondi, M. G. J. van der Burg, and A. W. K. Metzner, Proc. R. Soc. A 269, 21 (1962).

[36] E. Newman and R. Penrose, J. Math. Phys. (N.Y.) 3, 566 (1962).

[37] G. Barnich and C. Troessaert, Phys. Rev. Lett. 105, 111103 (2010).

[38] É. É. Flanagan and D. A. Nichols, Phys. Rev. D 95, 044002 (2017).

[39] J. Moxon, Companion package for "formulation and coordinates for the characteristic evolution system", https:// github.com/moxcodes/cce_formalism_companion.

[40] M. C. Babiuc, N. T. Bishop, B. Szilagyi, and J. Winicour, Phys. Rev. D 79, 084011 (2009).

[41] M. Campanelli and C. O. Lousto, Phys. Rev. D 59, 124022 (1999).

[42] L. Barack and A. Pound, Rep. Prog. Phys. 82, 016904 (2019).

[43] G. Barnich and C. Troessaert, J. High Energy Phys. 05 (2010) 062.

[44] M. Boyle et al., Classical Quantum Gravity 36, 195006 (2019).

[45] D. Iozzo, M. Boyle, S. Teukolsky et al. (to be published).

[46] M. Boyle, Phys. Rev. D 93, 084031 (2016).

[47] B. Szilagyi, Cauchy characteristic matching in general relativity, Ph.D. thesis, Pittsburgh University, 2000. 